WAGNeR, I. and N. F. WeEDEN (2000): Isozymes in Malus sylvestris, Malus domestica and in related Malus species. Acta Hort. 538, 51-56.

WAGner, I., H. P. Schmitt, W. Maurer and U. TABel (2004): Isozyme Polymorphism and Genetic Structure of Malus sylvestris (L.) MiLL. Native in Western Areas of Germany with Respect to Malus $\times$ domestica BoRKH. $\mathrm{XI}^{\text {th }}$ EUCARPIA Symp. On Fruit Breed. \& Genetics (Eds. F. Laurens und K. Evans) 1.-5.9.2003 in Angers. Acta Hort. 663, 545-550.

WEEDEN, N. F. (1984): Distinguishing among white seeded bean cultivars by means of allozyme genotypes. Euphytica 33, 199-208.
WeEDEN, N. F. and R. C. LAMB (1985): Identification of apple cultivars by isozyme phenotypes, J. Amer. Soc. Hort. Sci. 110, 509-515.

WeEden, N. F. and R. C. LAMB (1987): Genetics and linkage analysis of 19 isozyme loci in apple, J. Amer. Soc. Hort. Sci. 112, 865-872.

WENDEL, J. F. and N. F. WEEDEN (1989): Visualization and interpretation of plant isozymes, 5-45. In: Soltis, D. E.; Soltis, P. S. (eds.): Isozymes in plant biology, Chapman \& Hall, London.

ZANetto, A., G. Roussel and A. KREMER (1994): Geographic variation of inter-specific differentiation between Quercus robur L. and Quercus petraea (Matt.) Liebl. Forest Genetics 1, 111-123.

\title{
Comparative assessment of SSR, ISSR and AFLP markers for characterization of selected genotypes of Himalayan Chir pine (Pinus roxburghii Sarg.) based on resin yield
}

\author{
By A. RAWAT*'), S. BARThWAL and H. S. GinWAL \\ Division of Genetics and Tree Propagation, Forest Research Institute, P.O-I.P.E., \\ Kaulagarh Road, Dehradun-248195, Uttarakhand, India
}

(Received $11^{\text {th }}$ December 2013)

\begin{abstract}
A set of 19 SSR (Simple Sequence Repeats), 9 ISSR (Inter-Simple Sequence Repeats) and 5 AFLP (Amplified Fragment Length Polymorphism) primer combinations were used to evaluate the variability among 53 genotypes of Pinus roxburghii selected based on resin yield from the natural zone of occurrence of this species in Uttarakhand, India. The selected trees of pine varied in resin yield from 0.25 to $8 \mathrm{~kg} /$ year/tree. Based on the comparative assessment of SSR, ISSR and AFLP markers, SSR markers were found most polymorphic with an average PIC value of 0.327 and 2.42 alleles per marker, while ISSR markers showed the highest effective multiplex ratio (15.536) and marker index (4.958). AFLP markers showed the maximum resolving power (8.099) which was comparable to the resolving power (8.059) of ISSR markers. UPGMA-based dendrogram using SSR markers revealed more distinct grouping of genotypes on the basis of resin yield as compared to ISSR and AFLP markers. AMOVA by collection site revealed no significant variation among the populations. Whereas,
\end{abstract}

*) Author for correspondence: ANITA RAWAT. Ph: +91-9719408989, fax: +91-135-2756865. E-Mail: anitasrawat@gmail.com
AMOVA by resin yield using SSR, ISSR and AFLP markers revealed $\mathrm{F}_{S T}$ values to be $0.1096,0.0483$ and 0.2422 indicating moderate, low and great genetic differentiation among the groups. This clearly indicated that the variation at the molecular level was attributed to the resin yield and not the site of collection.

Key words: genetic diversity, fixation index, AMOVA, PIC.

\section{Introduction}

Pinus roxburghii Sarg. (Chir pine or long needle pine), is one of the most important conifers of northwestern Himalayas and is an important timber and resin yielding species. The forests of $P$. roxburghii are found ranging from longitudes $70^{\circ} \mathrm{E}$ to $93^{\circ} \mathrm{E}$ and latitudes $26^{\circ} \mathrm{N}$ to $36^{\circ} \mathrm{N}$ in subtropical and warm temperate monsoon belts, between 450 to $2300 \mathrm{~m}$ altitudes in Siwaliks and Himalayan main river valleys, from Kashmir to Bhutan. In India, Chir pine covers approximately $6,77,813$ ha area in the states of Himachal Pradesh, Jammu and Kashmir and Uttarakhand. Out of these three states, Uttarakhand alone contains a major portion of 4,12,000 ha of Chir pine (SINGH and KUMAR, 2004). Uttarakhand 
hills in the northern India are one of the best habitats for Chir pine forests.

Commercial Chir pine promotion in India was reported under the British regime during the $19^{\text {th }}$ century. Resin tapping for turpentine oil and timber for building railway sleepers were primary reasons for introducing Chir pine in the Himalayas (SINHA, 2002). Economically, pines are important source of wood, paper, resins, charcoal, food (particularly seeds), and ornamentals (LE MAITRE, 1998). The species is over exploited in Himalayan hills for timber and resin that cause considerable pressure on its forests. The conservationists are concerned on the damaging of pine forests due to large scale tapping of resins. Oleoresins from pines are composed of two components, volatile turpentine oil and the remaining solid transparent material called as Rosin. Turpentine oil finds its major use as a solvent in industries. It has medicinal qualities as well. It is used as an inhaler for nasal and throat ailments, applied to boils, heel cracks and on either side of the eye to reduce swelling (SingH et al., 1990). It is used in the manufacturing of lacquers and varnishes, synthesis of fragrant chemicals, insecticides and disinfectants. Rosin is the substance left after turpentine is removed. It is used in paper manufacturing, paper sizing, chemicals and pharmaceuticals, synthesis of ester gums and synthetic resins, paint, varnishes, printing inks, soap, rubber, surface coatings, floor coverings, adhesives, plastics etc. Resin is one of the most important non-wood forest products and $P$. roxburghii trees are known to yield the highest amount of oleoresin in India (COPPEN and HonE, 1995). In Uttarakhand, the tapping of resin began in 1890 that was commercialized in 1896. It was extended to Jammu and Kashmir and Himachal Pradesh in 1940 and 1945 respectively (SINGH and AsOKAN, 1984). Resin has got huge export potential. For the Himalayan region, extraction of resin as a source of revenue is reported since year 1911 (NAUTIYAL et al., 2006).

As per records (FAO, 1995), crude resin production peaked at about 75000 tonnes in 1975-76 and has since fallen steadily. Production in 1990-91 was less than 25000 tonnes, although it was then believed to have recovered and stabilized at about 25000-30000 tonnes (equivalent to approximately 18000-21000 tonnes of rosin). The main reason for the decline had been the loss of trees for tapping, either because many of them had reached the end of their productive lives and there were no new areas of pine with which to replace them, or because the damage done to trees by the use of inefficient, incorrectly applied methods of tapping had led to Forest Department bans on tapping. The loss of substantial indigenous production of crude resin and the demands of Indian industry for naval stores products have meant that India became a net importer of both rosin and turpentine. The shortfall in local production had been further compensated by the importation of about 10000 tonnes/year of crude resin. The major use of turpentine in India is for the production of synthetic camphor.

Diverse habitat of Chir pine in different geographical regions of Himalayas and Shivalik range supports the existence of natural variation. In order to increase the indigenous resin production in the country and generate a source of revenue, selections of pine trees have been made for growth and high resin yield under various tree improvement programs in the country (GUPTA et al., 1970; DOGRA, 1985; CHAUHAN and KANWAR, 2001; SHARMA et al., 2006).

Pines have long generation periods with vegetative phase extending more than 100 years and because of which multiple generations are not readily obtained and traditional approaches of tree improvement. The length of time needed for trees to reach reproductive maturity before controlled crosses is the limiting factor for tree improvement (MARTIN-TRILlO and MARTINEZ ZAPATER, 2002). Evaluation of variability in resin yield using molecular marker techniques could provide useful baseline information for the breeding programs. However, these molecular markers have technical differences in terms of cost, speed, amount of DNA needed, technical labor and degrees of polymorphism. Among the various molecular markers, polymerase chain reaction (PCR)-based markers, such as Random Amplified Polymorphic DNA (Williams et al., 1990), Inter Simple Sequence Repeat (ZIETKIEWIEZ et al., 1994), sequence-related amplified polymorphism (LI and QULROS, 2001) and Amplified Fragment Length Polymorphism (AFLP) (Vos et al., 1995) have become popular, as their application does not require any prior sequence information. On the other hand, microsatellite or Simple Sequence Repeat (SSR) are the markers of choice for breeding applications, but their development is more time consuming and expensive (GUPTA and VARSHNEY, 2000). However, the SSRs available in closely related species can be tested for trans-specific amplification thereby reducing both time and cost of development. To date, different marker systems have been applied for diversity analysis in $P$. roxburghii viz. Isozymes (Hussain, 1995; SHARMA, 1999; SHARMA, 2003), RAPD (MAlABADI and NATARAJA, 2007; GiNWAL et al., 2010), ISSR (MALABADI and NATARAJA, 2007; Parasharami and Thengane, 2012) and SSR (GAUli et al., 2009; CHAUHAN et al., 2010).

With the use of DNA based markers, there is a possibility to identify the high resin yielding genotypes at the nursery stage and that would help to establish plantations solely for the purpose of resin tapping. This will reduce the harm to naturally occurring forests of Chir pine and increase the resin production. But, before that there are certain questions to be answered such as whether the individuals varying in resin yield also vary in their genetic constitution and whether the variation at genetic level is responsible for the variation in resin yield. The analyses of polymorphisms using different molecular markers in genotypes of $P$. roxburghii varying in resin yield could help find answers to these questions. This study could be a preliminary step towards identification of markers for high resin yield that could be used further for Marker Assisted Selection of high resin yielding genotypes. Keeping in view, the above facts the objectives of the study presented in this paper were i) to characterize the genotypes of $P$. roxburghii varying in resin yield using three DNA based molecular markers (ISSR, SSR and AFLP) and ii) to compare the level of 
information provided by these three marker systems for evaluating the variability in resin yield and investigate the genetic basis of the trait.

\section{Materials and Methods}

The study was conducted in Western Himalayas in a naturally occurring Chir pine forest called Tiunee
(Chakrata range) in Uttarakhand state. The area is situated at an altitude of $1023 \mathrm{~m}$ with latitude $30^{\circ} 57^{\prime} 00^{\prime \prime} \mathrm{N}$ and longitude $77^{\circ} 51^{\prime} 00^{\prime \prime} \mathrm{E}$. The area enjoys moist temperate climate with an average annual rainfall of 1800 $\mathrm{mm}$ and mean temperature of $15^{\circ} \mathrm{C}$. Incidence of snowfall is moderate to heavy and restricted from December to February. The general forest type of the area is "Himalayan Moist Temperate" (SingHAL et al., 1987).

Table 1. - Geographical details of various samples of $P$. roxburghii.

\begin{tabular}{|c|c|c|c|c|c|c|c|c|}
\hline S. No. & Sample & Location & $\begin{array}{c}\text { Longitude } \\
\left({ }^{\circ} \mathrm{E}\right)\end{array}$ & $\begin{array}{c}\text { Latitude } \\
\left({ }^{\circ} \mathrm{N}\right)\end{array}$ & $\begin{array}{c}\text { Altitude } \\
\text { (m amsl)* }\end{array}$ & $\begin{array}{c}\text { Diameter } \\
(m)\end{array}$ & $\begin{array}{l}\text { Height } \\
\text { (m) }\end{array}$ & $\begin{array}{c}\text { Resin Yield } \\
\text { (kg/year) }\end{array}$ \\
\hline 1 & $\mathrm{~A}-01$ & Southern Aspect, Site Quality 01; Chatra & $77^{0} 56^{\prime} 16.9^{\prime \prime} \mathrm{E}$ & $30^{\circ} 58^{\prime} 02.3^{\prime \prime} \mathrm{N}$ & 1301 & 1.3 & 36.0 & 2.20 \\
\hline 2 & A-10 & Southerm Aspect, Site Quality 01; Chatra & $77^{0} 56^{\prime} 18.4^{\prime \prime} \mathrm{E}$ & $30^{\circ} 58^{\prime} \quad 02.2^{\prime \prime} \mathrm{N}$ & 1298 & 1.6 & 32.0 & 3.30 \\
\hline 3 & $\mathrm{~A}-12$ & Southern Aspect, Site Quality 01; Chatra & $77^{0} 56^{\prime} 42.9^{\prime \prime} \mathrm{E}$ & $30^{0} 56^{\prime} 54.9^{11} \mathrm{~N}$ & 1437 & 2.5 & 37.0 & 8.00 \\
\hline 4 & $A=13$ & Southern Aspect, Site Quality 01; Chatra & $77^{0} 56^{\prime} \quad 58.0^{\prime \prime} \mathrm{E}$ & $30^{\circ} 51^{\prime} 26.2^{\prime \prime} \mathrm{N}$ & 1203 & 1.8 & 24.8 & 3.35 \\
\hline 5 & A-19 & Southern Aspect, Site Quality 01; Chatra & $77^{0} 56^{\prime} \quad 17.4^{\prime \prime} \mathrm{E}$ & $30^{\omega} 58^{\prime} \quad 02.7^{\prime \prime} \mathrm{N}$ & 1307 & 1.5 & 35.0 & 5.20 \\
\hline 6 & A-02 & Southern Aspect, Site Quality 01; Chatra & $77^{0} 56^{\prime} 42.9^{\prime \prime} \mathrm{E}$ & $30^{\circ} 56^{\prime} 53.3^{\prime \prime} \mathrm{N}$ & 1423 & 2.3 & 37.0 & 6.50 \\
\hline 7 & A-24 & Southem Aspect, Site Quality 01; Chatra & $77^{0} 56^{\prime} \quad 20.3^{\prime \prime} \mathrm{E}$ & $30^{\circ} 58^{\prime} 01.0^{\prime \prime} \mathrm{N}$ & 1292 & 1.1 & 23.0 & 2.80 \\
\hline 8 & A-25 & Southern Aspect, Site Quality 01; Chatra & $77^{0} 56^{\prime} 20.3^{\prime \prime} \mathrm{E}$ & $30^{\circ} 58^{\prime} \quad 01.6^{\prime \prime} \mathrm{N}$ & 1292 & 1.5 & 24.5 & 2.90 \\
\hline 9 & A-28 & Southern Aspect, Site Quality 01; Chatra & $77^{0} 56^{\prime} 43.3^{\prime \prime} \mathrm{E}$ & $30^{\circ} 56^{\prime} 52.9^{\prime \prime} \mathrm{N}$ & 1421 & 1.1 & 29.0 & 6.20 \\
\hline 10 & $\mathrm{~A}-03$ & Southern Aspect, Site Quality 01; Chatra & $77^{\circ} 56^{\prime} 43.0^{\prime \prime} \mathrm{E}$ & $30^{\circ} 56^{\prime} 53.3^{\prime \prime} \mathrm{N}$ & 1423 & 1.7 & 36.8 & 0.90 \\
\hline 11 & A-06 & Southern Aspect, Site Quality 01; Chatra & $77^{0} 56^{\prime} \quad 43.2^{\prime \prime} \mathrm{E}$ & $30^{0} 56^{\prime} 53.6^{\prime \prime} \mathrm{N}$ & 1425 & 1.4 & 34.0 & 1.40 \\
\hline 12 & $\mathrm{~A}-07$ & Southern Aspect, Site Quality 01; Chatra & $77^{\circ} 56^{\prime} 18.0^{\prime \prime} \mathrm{E}$ & $30^{\circ} 68^{\prime} 03.3^{\prime \prime} \mathrm{N}$ & 1293 & 2.1 & 28.9 & 4.10 \\
\hline 13 & A-09 & Southern Aspect, Site Quality 01; Chatra & $77^{\circ} 56^{\prime} 42.5^{\prime \prime} \mathrm{E}$ & $30^{\circ} 56^{\prime} 54.4^{\prime \prime} \mathrm{N}$ & 1432 & 2.8 & 33.4 & 5.80 \\
\hline 14 & B-10 & Southerm Aspect, Site Quality 02; Chatra & $77^{0} 56^{\prime} 47.4^{\prime \prime} \mathrm{E}$ & $30^{\circ} 57^{\prime} 48.2^{\prime \prime} \mathrm{N}$ & 1454 & 2.6 & 27.0 & 2.00 \\
\hline 15 & B-12 & Southern Aspect, Site Quality 02; Chatra & $77^{0} 56^{\prime} 48.4^{1} \mathrm{E}$ & $30^{\circ} 56^{\prime} \quad 49.3^{\prime \prime} \mathrm{N}$ & 1451 & 1.1 & 28.4 & 4.60 \\
\hline 16 & B-13 & Southern Aspect, Site Quality 02; Chatra & $77^{0} 56^{\prime} 46.3^{\prime \prime} \mathrm{E}$ & $30^{\circ} 56^{\prime} 42.4^{\prime \prime} \mathrm{N}$ & 1443 & 1.8 & 28.0 & 3.60 \\
\hline 17 & B-14 & Southern Aspect, Site Quality 02; Chatra & $77^{\circ} 56^{\prime} \quad 47.6^{\prime \prime} \mathrm{E}$ & $30^{0} 56^{\prime} 49.3^{\prime \prime} \mathrm{N}$ & 1442 & 2.5 & 28.0 & 0.25 \\
\hline 18 & B-18 & Southern Aspect, Site Quality 02; Chatra & $77^{0} 56^{\prime} \quad 28.4^{\prime \prime} \mathrm{E}$ & $30^{\circ} 57^{\prime} 53.7^{\prime \prime} \mathrm{N}$ & 1302 & 2.1 & 45.0 & 3.35 \\
\hline 19 & B-19 & Southern Aspect, Site Quality 02; Chatra & $77^{\circ} 56^{\prime} \quad 22.1^{\prime \prime} \mathrm{E}$ & $30^{0} 57^{\prime} 57.6^{11} \mathrm{~N}$ & 1315 & 1.4 & 37.0 & 2.70 \\
\hline 20 & B-02 & Southern Aspect, Site Quality 02; Chatra & $77^{\circ} 56^{\prime} 46.4^{11 E} \mathrm{E}$ & $30^{\circ} 57^{\prime} 47.5^{\prime \prime} \mathrm{N}$ & 1450 & 2.5 & 29.2 & 2.50 \\
\hline 21 & B-24 & Southern Aspect, Site Quality 02; Chatra & $77^{0} 56^{\prime} 46.6^{\prime \prime} \mathrm{E}$ & $30^{\circ} 56^{\prime} 47.1 " \mathrm{~N}$ & 1438 & 2.5 & 28.0 & 5.70 \\
\hline 22 & B-25 & Southem Aspect, Site Quality 02; Chatra & $77^{0} 56^{\prime} 46.8^{\prime \prime} \mathrm{E}$ & $30^{\circ} 56^{\prime} 42.2^{\prime \prime} \mathrm{N}$ & 1437 & 1.4 & 34.0 & 6.40 \\
\hline 23 & $\mathrm{~B}-26$ & Southern Aspect, Site Quality 02; Chatra & $77^{0} 56^{\prime} \quad 21.7^{\prime \prime} \mathrm{E}$ & $30^{\circ} 57^{\prime} 58.3^{\prime \prime} \mathrm{N}$ & 1306 & 1.3 & 28.0 & 2.80 \\
\hline 24 & B-03 & Southern Aspect, Site Quality 02; Chatra & $77^{0} 56^{\prime} \quad 46.7^{\prime \prime} \mathrm{E}$ & $30^{\circ} 56^{\prime} 47.4^{\prime \prime} \mathrm{N}$ & 1450 & 2.6 & 29.0 & 4.90 \\
\hline 25 & B-04 & Southern Aspect, Site Quality 02; Chatra & $77^{\circ} 56^{\prime} \quad 22.7^{\prime \prime E}$ & $30^{\circ} 57^{\prime} 58.0^{\prime \prime} \mathrm{N}$ & 1316 & 2.3 & 30.0 & 0.80 \\
\hline 26 & B-06 & Southern Aspect, Site Quality 02; Chatra & $77^{\circ} 56^{\prime} 47.0^{\prime \prime} \mathrm{E}$ & $30^{\circ} 56^{\prime} 47.3^{\prime \prime} \mathrm{N}$ & 1452 & 1.4 & 31.0 & 4.50 \\
\hline 27 & $\mathrm{~B}-07$ & Southern Aspect, Site Quality 02; Chatra & $77^{0} 56^{\prime} 21.6^{\prime \prime} \mathrm{E}$ & $30^{0} 57^{\prime} 58.0^{\prime \prime} \mathrm{N}$ & 1324 & 2.1 & 30.0 & 4.30 \\
\hline 28 & B-08 & Southern Aspect, Site Quality 02; Chatra & $77^{0} 56^{\prime} 47.3^{\prime \prime} \mathrm{E}$ & $30^{\circ} 57^{\prime} 48.5^{\prime \prime} \mathrm{N}$ & 1462 & 1.5 & 27.0 & 2.25 \\
\hline 29 & B-09 & Southern Aspect, Site Quality 02; Chatra & $77^{0} 56^{\prime} \quad 47.0^{\prime \prime} \mathrm{E}$ & $30^{\circ} 56^{\prime} 48.3^{\prime \prime} \mathrm{N}$ & 1454 & 2.2 & 27.0 & 4.70 \\
\hline 30 & $C-01$ & Northern Aspect, Site Quality 01; Chatra & $77^{0} 56^{\prime} 36.5^{\prime \prime} \mathrm{E}$ & $30^{0} 57^{\prime} 09.8^{\prime \prime} \mathrm{N}$ & 1379 & 1.5 & 35.0 & 4.20 \\
\hline 31 & $C-10$ & Northern Aspect, Site Quality 01; Chatra & $77^{0} 56^{\prime} 48.6^{\prime \prime} \mathrm{E}$ & $30^{\circ} 56^{\prime} 47.9^{\prime \prime} \mathrm{N}$ & 1406 & 1.5 & 34.0 & 2.25 \\
\hline 32 & $c-12$ & Northern Aspect, Site Quality 01; Chatra & $77^{0} 56^{\prime} 37.2^{\prime \prime} \mathrm{E}$ & $30^{\circ} 57^{\prime} 10.1^{\prime \prime} \mathrm{N}$ & 1364 & 2.9 & 36.0 & 2.25 \\
\hline 33 & $C-15$ & Northern Aspect, Site Quality 01; Chatra & $77^{\circ} 56^{\prime} 47.7^{\prime \prime} \mathrm{E}$ & $30^{\circ} 56^{\prime} 49.3^{\prime \prime} \mathrm{N}$ & 1404 & 1.8 & 35.0 & 2.90 \\
\hline 34 & $c-20$ & Northern Aspect, Site Quality 01; Chatra & $77^{0} 56^{\prime} 46.6^{\prime \prime} \mathrm{E}$ & $30^{n} 56^{\prime} 50.3^{\prime \prime} \mathrm{N}$ & 1401 & 1.5 & 32.0 & 2.90 \\
\hline 35 & $\mathrm{C}-03$ & Northern Aspect, Site Quality 01; Chatra & $77^{0} 56^{\prime} 47.8^{\prime \prime} \mathrm{E}$ & $30^{\circ} 56^{\prime} 46.1^{11} \mathrm{~N}$ & 1404 & 2.5 & 40.0 & 5.00 \\
\hline 36 & $\mathrm{C}-04$ & Northern Aspect, Site Quality 01; Chatra & $77^{0} 56^{\prime} \quad 37.2^{\prime \prime} \mathrm{E}$ & $30^{0} 57^{\prime} \quad 09.8^{\prime \prime} \mathrm{N}$ & 1379 & 1.9 & 42.8 & 2.70 \\
\hline 37 & C-07 & Northern Aspect, Site Quality 01; Chatra & $77^{0} 56^{\prime} 48.4^{\prime \prime E}$ & $30^{\circ} 56^{\prime} 47.7^{\prime \prime} \mathrm{N}$ & 1404 & 2.5 & 38.0 & 4.50 \\
\hline 38 & C.08 & Northern Aspect, Site Quality 01; Chatra & $77^{\circ} 56^{\prime} 38.8^{\prime \prime} \mathrm{E}$ & $30^{0} 57^{\prime} 11.2^{\mathrm{N}} \mathrm{N}$ & 1360 & 1.2 & 36.0 & 4.00 \\
\hline 39 & 0.09 & Northern Aspect, Site Quality 01; Chatra & $77^{0} 56^{\prime} 49.5^{\prime \prime} \mathrm{E}$ & $30^{\circ} 56^{\prime} 47.5^{\prime \prime} \mathrm{N}$ & 1405 & 2.0 & 39.0 & 5.60 \\
\hline 40 & D-11 & Northern Aspect, Site Quality 02; Chatra & $77^{0} 56^{\prime} \quad 30.4^{4 \prime} \mathrm{E}$ & $30^{\circ} 58^{\prime} \quad 04.9^{\prime \prime} \mathrm{N}$ & 1171 & 1.6 & 29.5 & 2.60 \\
\hline 41 & D-14 & Northern Aspect, Site Quality 02; Chatra & $77^{0} 56^{\prime} 31.0^{\prime \prime} \mathrm{E}$ & $30^{\circ} 58^{\prime} \quad 05.1^{\prime \prime N}$ & 1171 & 2.5 & 36.6 & 2.30 \\
\hline 42 & D-24 & Northem Aspect, Site Quality 02; Chatra & $77^{\circ} 56^{\prime} 37.0^{\prime \prime} \mathrm{E}$ & $30^{\circ} 58^{\prime} 06.3^{\prime \prime} \mathrm{N}$ & 1160 & 1.4 & 29.2 & 1.75 \\
\hline 43 & $\mathrm{D}-26$ & Northern Aspect, Site Quality 02; Chatra & $77^{0} 56^{\prime} 35.5{ }^{\prime \prime E}$ & $30^{0} 57^{\prime} 03.2^{\prime \prime} \mathrm{N}$ & 1352 & 2.2 & 34.2 & 1.80 \\
\hline 44 & $\mathrm{D}-27$ & Northern Aspect, Site Quality 02; Chatra & $77^{0} 56^{\prime} 38.2^{\prime \prime} \mathrm{E}$ & $30^{\circ} 57^{\prime} 04.4^{\prime \prime} \mathrm{N}$ & 1360 & 1.3 & 36.0 & 0.90 \\
\hline 45 & D-29 & Northern Aspect, Site Quality 02; Chatra & $77^{\circ} 56^{\prime} 38.0^{\prime \prime} \mathrm{E}$ & $30^{\circ} 57^{\prime} 06.2^{\prime \prime} \mathrm{N}$ & 1353 & 1.3 & 27.9 & 2.10 \\
\hline 46 & D-30 & Northern Aspect, Site Quality 02; Chatra & $77^{0} 56^{\prime} 38.3^{\prime \prime} \mathrm{E}$ & $30^{\circ} 57^{\prime} 06.5^{\prime \prime} \mathrm{N}$ & 1355 & 2.2 & 40.2 & 1.60 \\
\hline 47 & D-31 & Northern Aspect, Site Quality 02; Chatra & $77^{0} 56^{\prime} \quad 43.7^{\prime \prime} \mathrm{E}$ & $30^{\circ} 56^{\prime} 53.9^{\prime \prime} \mathrm{N}$ & 1396 & 1.5 & 35.0 & 4.20 \\
\hline 48 & D-33 & Northern Aspect, Site Quality 02; Chatra & $77^{0} 56^{\prime} 42.4^{\prime \prime} \mathrm{E}$ & $30^{0} 56^{\prime} 53.5^{\prime \prime} \mathrm{N}$ & 1393 & 2.5 & 40.0 & 5.00 \\
\hline 49 & D-37 & Northern Aspect, Site Quality 02; Chatra & $77^{\circ} 56^{\prime} \quad 42.4^{\prime \prime} \mathrm{E}$ & $30^{\circ} 56^{\prime} 54.1^{\prime \prime} \mathrm{N}$ & 1432 & 2.5 & 38.0 & 4.50 \\
\hline 50 & D-38 & Northern Aspect, Site Quality 02; Chatra & $77^{0} 56^{\prime} \quad 43.2^{\prime \prime} \mathrm{E}$ & $30^{\circ} 56^{\prime} 53.4^{\prime \prime} \mathrm{N}$ & 1423 & 1.2 & 36.0 & 4.00 \\
\hline 51 & D-39 & Northern Aspect, Site Quality 02; Chatra & $77^{\circ} 56^{\prime} 42.2^{\prime \prime} \mathrm{E}$ & $30^{\circ} 56^{\prime} 54.3^{\prime \prime} \mathrm{N}$ & 1421 & 2.0 & 39.0 & 5.60 \\
\hline 52 & $\mathrm{D}-07$ & Northern Aspect, Site Quality 02; Chatra & $77^{\circ} 56^{\prime} 38.8^{\prime \prime} \mathrm{E}$ & $30^{\circ} 57^{\prime} \quad 04.5^{\prime \prime} \mathrm{N}$ & 1370 & 1.6 & 28.3 & 1.30 \\
\hline 53 & D-08 & Northern Aspect, Site Quality 02; Chatra & $77^{\circ} 56^{\prime} 41.8^{\prime \prime} \mathrm{E}$ & $30^{\circ} 57^{\prime} 07.2^{\prime \prime} \mathrm{N}$ & 1365 & 1.8 & 29.2 & 1.10 \\
\hline
\end{tabular}

* amsl-above mean sea level. 


\section{Plant material}

A total of 240 genotypes of Chir pine were evaluated for resin yield in a study conducted by Forest Research Institute, Dehradun. The study revealed non-significant correlation of tree diameter, altitude and site quality with resin yield in Uttarakhand. Based on these observations, Chakrata division (Uttarakhand) having a large natural population of Chir pine was selected for carrying out molecular characterization work and association analysis for identification of trait specific DNA markers. The experiment was laid in the natural forest of Chir pine at an altitude ranging from 1000 to $1500 \mathrm{~m}$ above the mean sea level covering southern aspect (A1) and northern aspect (A2), each with two sites having different site qualities (S1 and S2). The site quality was adjudged as per the height of dominant trees or slopes. For each site quality, three plots ( 0.25 ha each) were selected at random, comprising total area of 0.75 ha. The plots were considered as replications. For each replication, entire area of 0.25 ha was surveyed for collection of data. For obtaining precise information, boundary trees were ignored. Geographical details of the samples along with the phenotypic data of resin yield of these genotypes is presented in Table 1. Young needles or sapwood (in case needles were not available due to extreme height of trees) samples were collected from the site and stored at $-80^{\circ} \mathrm{C}$.

\section{DNA extraction and quantification}

DNA was extracted from young needles using a combination of the methods described by STANGE et al. (1998) and Doyle and Doyle (1990) and from the sapwood following a combination of the protocols given by ASIF and CAnnon (2005) and Doyle and Doyle (1990). The quality of DNA was tested on $0.8 \%$ agarose gel and the DNA concentration was quantified using BioPhotometer (Eppendrof 6131, Germany). DNA samples were diluted to the required concentration $(15 \mathrm{ng} / \mu \mathrm{l}$ for ISSR and SSR analysis and $250 \mathrm{ng} / \mathrm{\mu l}$ for AFLP analysis) for polymerase chain reaction (PCR) amplification.

\section{SSR analysis}

A total of 80 SSR markers (47 nuclear SSRs and 33 chloroplast SSRs) from different species of pines (nuclear SSRs were from $P$. resinosa, $P$. taeda, $P$. merkussi and $P$. densiflora whereas, chloroplast SSRs were from $P$. thunbergii and $P$. sylvestris) were screened for amplification in $P$. roxburghii. Out of these, 48 SSRs showed positive amplification and $19 \mathrm{SSR}$ sere used in the present study based on the polymorphism (Table 2). PCR was performed in a $15 \mu$ reaction volume (VENDRAMIN et al., 1996) containing $15 \mathrm{ng}$ of template DNA, 1X Taq buffer, $3.0 \mathrm{mM} \mathrm{MgCl} 2,0.2 \mathrm{mM}$ dNTPs, $0.2 \mu \mathrm{M}$ of each primer and $0.06 \mathrm{U}$ of Taq DNA polymerase (Bangalore Genei Pvt. Ltd., India). PCR amplification was car-

Table 2. - Characteristics of SSR loci, including their repeat motif, the number of alleles per locus, number of polymorphic alleles and percent polymorphism.

\begin{tabular}{|c|c|c|c|c|c|c|c|c|c|c|c|}
\hline S. No. & Locus & & Primer Sequence $\left(5^{\prime \prime}-3^{\prime}\right)$ & $\begin{array}{l}\mathrm{Ta} \\
\left({ }^{\circ} \mathrm{C}\right) \\
\end{array}$ & $\begin{array}{c}\text { Sixe range } \\
\text { (bp) }\end{array}$ & Repent motif & PIC & $\begin{array}{l}\text { No. of } \\
\text { illeles }\end{array}$ & $\begin{array}{l}\text { polymorphis } \\
\text { alleles }\end{array}$ & $\begin{array}{c}\% \\
\% \\
\text { palymorphism }\end{array}$ & Reference \\
\hline 1 & pdme 011 & $\begin{array}{l}\text { Forward } \\
\text { Reverse }\end{array}$ & $\begin{array}{l}\text { TGTCAACTATATGG TACOANAC } \\
\text { CCICANTGAICAMAICC }\end{array}$ & 55 & $1 \cdot 11-147$ & $(\mathrm{GT})_{16}$ & 0.153 & 2 & 1 & 50 & $\begin{array}{c}\text { Watunabe et al. } \\
2006\end{array}$ \\
\hline 2 & pdins 221 & $\begin{array}{l}\text { Forward } \\
\text { Reverse }\end{array}$ & $\begin{array}{l}\text { GACAACTTGATGACGGAAATAC } \\
\text { CCAACACAAAAGTGTACTTC }\end{array}$ & 55 & $151-201$ & $(G A) G(G A) s$ & 0.115 & 2 & 1 & 50 & $\begin{array}{l}\text { Watanabe ct al. } \\
2006\end{array}$ \\
\hline 3 & $\sin 0 s$ & $\begin{array}{l}\text { linuard } \\
\text { Rutersce }\end{array}$ & $\begin{array}{l}\text { GACTCTANTTCANACCCCA } \\
\text { TOCAOATCTACCACTTTTTC }\end{array}$ & 60 & $98-120$ & $(\mathrm{lGl}$ & 0.376 & 2 & 2 & 100 & $\begin{array}{l}\text { Niutjulajaringsih } \\
\text { ot al.. 2005 }\end{array}$ \\
\hline 4 & $\mathrm{pm} 07$ & $\begin{array}{l}\text { Forwated } \\
\text { Rewerse }\end{array}$ & $\begin{array}{l}\text { GAMTCTAMGCATATGAMTGAG } \\
\text { CTTGTTANTGCTACTATTATG }\end{array}$ & $5 \overline{5}$ & $190-230$ & $(\mathrm{AC}) \mathrm{A} / \mathrm{AT}$ & 4).211 & 2 & 1 & 50 & $\begin{array}{c}\text { Nurtjahjaringsih } \\
\text { et al.. 2005 }\end{array}$ \\
\hline 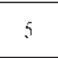 & PITX 3025 & $\begin{array}{l}\text { Furkard } \\
\text { Riverse }\end{array}$ & $\begin{array}{l}\text { CACGCIGIALAATAACANTCL } \\
\text { TTCTATATCGCTTTTAGTTTC }\end{array}$ & 55 & $273-290$ & $(C A)_{11}$ & 0.497 & 2 & 1 & 50 & Elsik ut al., Z Z(100) \\
\hline 6 & RPTesto & $\begin{array}{l}\text { Forkard } \\
\text { Reverse }\end{array}$ & $\begin{array}{l}\text { AGGAICCAACAGCAICACC } \\
\text { CIGAACATGAAGGCAGIGI }\end{array}$ & 60 & $73-77$ & $\mathrm{Toc}$ & 0.164 & 2 & 1 & 50 & $\begin{array}{l}\text { Chasne et al., } \\
2004\end{array}$ \\
\hline 7 & RP'Tste & $\begin{array}{l}\text { Fornard } \\
\text { Rererse }\end{array}$ & $\begin{array}{l}\text { CCAGACAACCCAAATCAACIG } \\
\text { GCCIGCTAICGMACCACAA }\end{array}$ & 60 & $314-357$ & $A G C$ & 0.100 & 2 & 2 & 100 & $\begin{array}{l}\text { (hagne et al., } \\
2004\end{array}$ \\
\hline 8 & {$[3+1254$} & $\begin{array}{l}\text { l'orward } \\
\text { lRewerse }\end{array}$ & $\begin{array}{l}\text { CAAITGGAATGAGAACAGATAGG } \\
\text { TGCGTTGCACTTCGTATAG }\end{array}$ & $\$ 7.8$ & $146-161$ & $(T)_{1} ?$ & 0.414 & 2 & 2 & 100 & $\begin{array}{c}\text { Vendramin et al.. } \\
1996\end{array}$ \\
\hline 9 & {$[17] 936$} & $\begin{array}{l}\text { Fowatd } \\
\text { Reverse }\end{array}$ & $\begin{array}{l}\text { TTCATTGGAAATACACTAGCCC } \\
\text { DAAACCGIACACGAGATICCC }\end{array}$ & 55 & $141-143$ & $\left(\mathrm{~T}_{16}\right.$ & 0.499 & 2 & 2 & 100 & $\begin{array}{c}\text { Vendramin er al., } \\
1906\end{array}$ \\
\hline 10 & PL87268 & $\begin{array}{l}\text { Fonvard } \\
\text { Reverse }\end{array}$ & $\begin{array}{l}\text { GCCAGGGAAAATCGTAGG } \\
\text { AGACGATAGACATCCAACC }\end{array}$ & 55 & $138-182$ & ('l his & 0.324 & 3 & 3 & 100 & $\begin{array}{c}\text { Vendramin er al } \\
1996\end{array}$ \\
\hline 11 & pm $09 \mathrm{a}$ & $\begin{array}{l}\text { Forward } \\
\text { Rewerse }\end{array}$ & $\begin{array}{l}\text { COITCICATTICGNTATGCAC } \\
\text { AIIAAAGGIALAKGGGC }\end{array}$ & 58.5 & $108-115$ & $(A T)=(G T) \&(A T):$ & 0.339 & 3 & 3 & 100 & $\begin{array}{l}\text { Rurtjahijaringsili } \\
\text { at al., } 2005\end{array}$ \\
\hline 12 & PCP26106 & $\begin{array}{l}\text { Forward } \\
\text { Reverse }\end{array}$ & $\begin{array}{l}\text { AATCCGACAAAAAAGATTCGG } \\
\text { GCICCATTCACGTGGTTS }\end{array}$ & 58.5 & $144-155$ & $(A): A$ & 0.348 & 3 & 3 & 100 & Provaln et al., 1998 \\
\hline 13 & $\mathrm{PCP} 30277$ & $\begin{array}{l}\text { linward } \\
\text { Reversic }\end{array}$ & $\begin{array}{l}\text { TGITCAICICGIAGCGGMAG } \\
\text { ATCMATCANTCACTCCOC }\end{array}$ & 58.5 & $124-182$ & $(A) \ldots(G)$ & 0.391 & 2 & 2 & 100 & 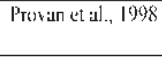 \\
\hline 14 & P1302014 & $\begin{array}{l}\text { Forward } \\
\text { Ruwerse }\end{array}$ & $\begin{array}{l}\text { TCATAGCGGAAGATCCTCTTT } \\
\text { CGGATTGATCCTMACATACC }\end{array}$ & 36 & $143-145$ & 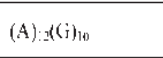 & 0.292 & 4 & 4 & 100 & $\begin{array}{c}\text { Vendranim et at.". } \\
1990\end{array}$ \\
\hline 15 & $P(45002$ & $\begin{array}{l}\text { Forward } \\
\text { Rewerse }\end{array}$ & 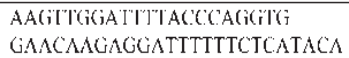 & 58 & $1,54-165$ & $(T)_{1:}$ & 0.262 & 5 & 4 & 80 & $\begin{array}{c}\text { Vendfarritit er al } \\
1996\end{array}$ \\
\hline 16 & {$[779951$} & $\begin{array}{l}\text { lourard } \\
\text { Reverse }\end{array}$ & $\begin{array}{l}\text { CITTTGTITTCAACAATGCA } \\
\text { ACATCLATCTCCATACGGC }\end{array}$ & 585 & $124-126$ & $\left(\mathrm{~T}_{1}:\right.$ & 0. 459 & 2 & 2 & 100 & $\begin{array}{l}\text { Vendramin et al., } \\
1996\end{array}$ \\
\hline 17 & $\operatorname{PCP} 9434$ & $\begin{array}{l}\text { Fonkard } \\
\text { Reterse }\end{array}$ & $\begin{array}{l}\text { AMATCACGTAGATGOCATGG } \\
\text { GCGGIAIGAGGGAAGAAGC }\end{array}$ & 58.5 & $114-117$ & $(A): 0$ & 0.486 & 2 & 2 & 100 & Ptovan atal., 1498 \\
\hline 18 & $\mathrm{Pt} 36480$ & $\begin{array}{l}\text { Finwatd } \\
\text { Reversie }\end{array}$ & $\begin{array}{l}\text { TT"TGGCTTACAAAATAAAAGAGC } \\
\text { AAAT"TC CIAAAGAAGI IAAGAGCACA }\end{array}$ & 52 & $137-145$ & $(T)_{11}$ & 0.303 & 2 & 2 & 100 & $\begin{array}{c}\text { Vendramin stat, } \\
1996\end{array}$ \\
\hline 19 & $[\mathrm{CP} 4] 13]$ & $\begin{array}{l}\text { Forward } \\
\text { Reverse }\end{array}$ & $\begin{array}{l}\text { AMAGCATITCCAGITGGGG } \\
\text { GGICAGGAIICA]GIICLICC }\end{array}$ & 58.5 & $137-142$ & $\left(T_{41}\right.$ & 0.473 & 2 & 2 & 100 & PTevan ęla al., 1998 \\
\hline
\end{tabular}


Table 3. - Details of ISSR analysis, including the total number of bands, polymorphic bands and percent polymorphism.

\begin{tabular}{|c|c|c|c|c|c|c|c|}
\hline S.No. & Primer & Base Sequence $\left(5^{\prime}-3^{\prime}\right)$ & $\mathrm{T}_{2}\left({ }^{\circ} \mathrm{C}\right)$ & PIC & $\begin{array}{c}\text { Total no. of } \\
\text { bands }\end{array}$ & $\begin{array}{c}\text { Polymorphic } \\
\text { bands }\end{array}$ & $\%$ polymorphism \\
\hline 1 & $11 B C 809$ & GAGGAG $\wedge \mathrm{GAG} \triangle \mathrm{M} \wedge \mathrm{G} \wedge \mathrm{GG}$ & 57.7 & 0.321 & 17 & 14 & 82 \\
\hline 2 & UBC 820 & GlGLGIGIGlGlGlGlC & 56.8 & 0.272 & 8 & 5 & 63 \\
\hline 4 & UBC 880 & GGAGAGGAGAGGAGA & 48.4 & 0.064 & 9 & 3 & 33 \\
\hline 5 & UBCXOS & AGAGAGAGAGAGAGAGG & 56.8 & 0.340 & $n$ & 7 & 48 \\
\hline 6 & [UBC8]1 & GAGAGAGAGAGAGAGAC & 50.0 & 0.326 & 22 & 22 & 100 \\
\hline 9 & UBC 873 & GACAG $\Lambda C A G A C A G \wedge C A$ & 51.6 & 0.322 & 28 & 28 & 100 \\
\hline
\end{tabular}

Table 4. - Details of AFLP analysis, including the total number of bands, polymorphic bands and percent polymorphism.

\begin{tabular}{|c|c|c|c|c|c|}
\hline S.No. & Primcr combination & PIC & Total no. of bands & Polymorphic bands & $\%$ polymorphism \\
\hline 1 & $\mathrm{E}_{\mathrm{ASA}} \mathrm{M}_{\mathrm{C} \mathrm{KSi}}$ & 0.196 & 75 & 43 & 57 \\
\hline 3 & $E_{\text {Ka: }} M_{C: k, i 1}$ & 0.053 & 44 & 10 & 2.3 \\
\hline 5 & $\mathrm{~L}_{\mathrm{AAG}} \mathrm{M}_{\mathrm{CTAC}}$ & 0.045 & 28 & 6 & 2] \\
\hline
\end{tabular}

ried out at $5 \mathrm{~min}$. at $95^{\circ} \mathrm{C}$ followed by 30 cycles of $1 \mathrm{~min}$. at $94^{\circ} \mathrm{C}, 1 \mathrm{~min}$. at $55^{\circ} \mathrm{C}$ to $60^{\circ} \mathrm{C}$ (as per the annealing temperature of the primer) and $1 \mathrm{~min}$. at $72^{\circ} \mathrm{C}$ and a final extension of $8 \mathrm{~min}$. at $72^{\circ} \mathrm{C}$. Amplified products were electrophoresed on $3 \%(\mathrm{w} / \mathrm{v})$ metaphor agarose: agarose (3:1) gel with $1 \mathrm{X}$ TBE buffer and stained with ethidium bromide $(0.5 \mu \mathrm{g} / \mathrm{ml})$. DNA fragments were visualized under UV light and documented with the gel documentation imaging system (GelDoc-It System, UVP Ltd.). The primers which were not resolved on metaphor-agarose gel were then separated on $8 \%(\mathrm{w} / \mathrm{v})$ polyacrylamide gel casted in 'MEGA-GEL High Throughput Vertical Unit' (model C-DASG-400-50) marketed by C.B.S Scientific Co. (Del Mar, CA, USA) with 1X TBE buffer. DNA fragments were visualized under UV light (ULTRA Lum Inc) and photographed with digital camera (Cannon, EOS 400D).

\section{ISSR analysis}

Twenty five ISSR primers (University of British Columbia (UBC), Biotechnology laboratory, Vancouver, BC, Canada) were initially screened for amplification. Based on polymorphism, finally 9 ISSR markers were used to conduct the present study (Table 3). PCR was performed in a $20 \mu \mathrm{l}$ reaction volume (HoNG et al., 2007) containing $15 \mathrm{ng}$ of template DNA, 1X Taq buffer, $1.75 \mathrm{mM} \mathrm{MgCl}_{2}, 0.2 \mathrm{mM}$ dNTPs, $0.4 \mu \mathrm{M}$ primer and $0.6 \mathrm{U}$ of Taq DNA polymerase (Bangalore Genei Pvt. Ltd., Bangalore, India). PCR amplification was carried out at $5 \mathrm{~min}$. at $94^{\circ} \mathrm{C}$ followed by 40 cycles of $30 \mathrm{sec}$. at $94^{\circ} \mathrm{C}, 30$ sec. at annealing temperature (primer specific) and $1 \mathrm{~min}$. at $72^{\circ} \mathrm{C}$ and a final extension of $10 \mathrm{~min}$. at $72^{\circ} \mathrm{C}$. Amplified products were electrophoresed on $2 \%$ (w/v) agarose gel with $1 \mathrm{X}$ TBE buffer. DNA fragments were visualized under UV light and documented with the gel documentation imaging system (GelDoc-It System, UVP Ltd.).

\section{AFLP analysis}

A total of 12 primer combinations (Table 4) were screened for amplification, out of which 5 primer combinations were found to be polymorphic. AFLP was carried out using the standard protocol described by Vos et al. (1995) using a commercial kit (Invitrogen) using $250 \mathrm{ng}$ DNA of each accession. All PCR reactions were performed in a Gene Amp PCR 9700 Thermal Cycler. The samples were size-fractionated on $6 \%$ polyacrylamide gels using Sequigen GT (Bio-Rad, Hercules, USA) under denaturing conditions. The fragments were detected by autoradiography.

\section{Gel scoring and data analysis}

The profiles produced by ISSR, SSR and AFLP markers were scored manually: each allele was scored as present (1) or absent (0) for each of the ISSR, SSR and AFLP loci.

Genotyping data obtained for the SSR, ISSR and AFLP markers was used to assess the discriminatory power of the markers by evaluating four parameters: Polymorphism Information Content (PIC), Effective Multiplex Ratio (EMR), Marker Index (MI) and Resolving Power (RP). The PIC value provides an estimate of the discriminating power of a marker. Polymorphic information content was calculated using the formula given by ROLDAN-RUIZ et al. (2000)

$$
\mathrm{PICi}=\left[2 \mathrm{f}_{\mathrm{i}}\left(1-\mathrm{f}_{\mathrm{i}}\right)\right]
$$

Where, PICi is the polymorphism information content of marker $i, f_{i}$ is the frequency of the marker fragments 
which were present and $1-f_{i}$ the frequency of marker fragments which were absent. PIC was averaged over the fragments for each primer combination.

The average number of DNA fragments amplified/detected per genotype using a marker system is considered as multiplex ratio $(n)$. The number of loci polymorphic in the germplasm set of interest, analyzed per experiment, called effective multiplex ratio (EMR) was calculated by following the formula described by VARSHNEY et al. (2007)

\section{$\mathrm{EMR}=\mathrm{n} \cdot \beta$}

Where, $\mathrm{n}$ is the total number of loci and $\beta$ is the fraction of polymorphic markers which is estimated after considering the polymorphic loci (np) as $\beta=n p /$ $(\mathrm{np}+\mathrm{nnp})$
The utility of a given marker system is a balance between the level of polymorphism detected and the extent to which an assay can identify multiple polymorphisms. A product of information content, as measured by PIC, and effective multiplex ratio, called as marker index may provide a convenient estimate of marker utility POWELL et al. (1996)

\section{$\mathrm{MI}=\mathrm{PIC} \times \mathrm{EMR}$}

Resolving power of each primer was calculated according to PREvost and WiLKinson (1999).

$$
\mathrm{RP}=\Sigma \mathrm{Ib}
$$

where, Ib represents fragment informativeness. The Ib can be represented into a $0-1$ scale by the following formula:

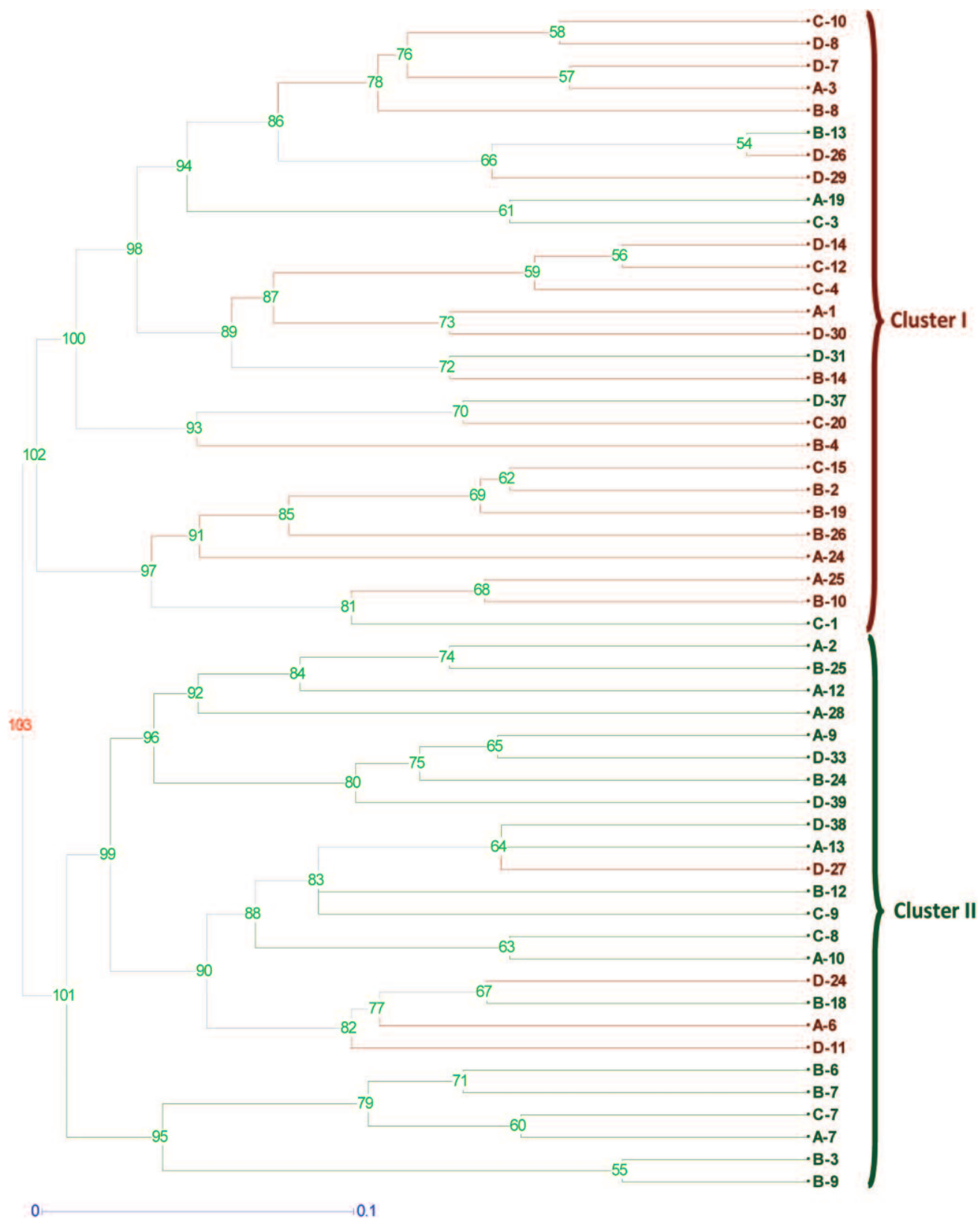

Figure 1. - Dendrogram showing genetic relationship among $P$. roxburghii genotypes varying in resin yield using SSR markers. 
SI 1. - Genetic dissimilarity index between high and low resin yielding genotypes of $P$. roxburghii using SSR markers.

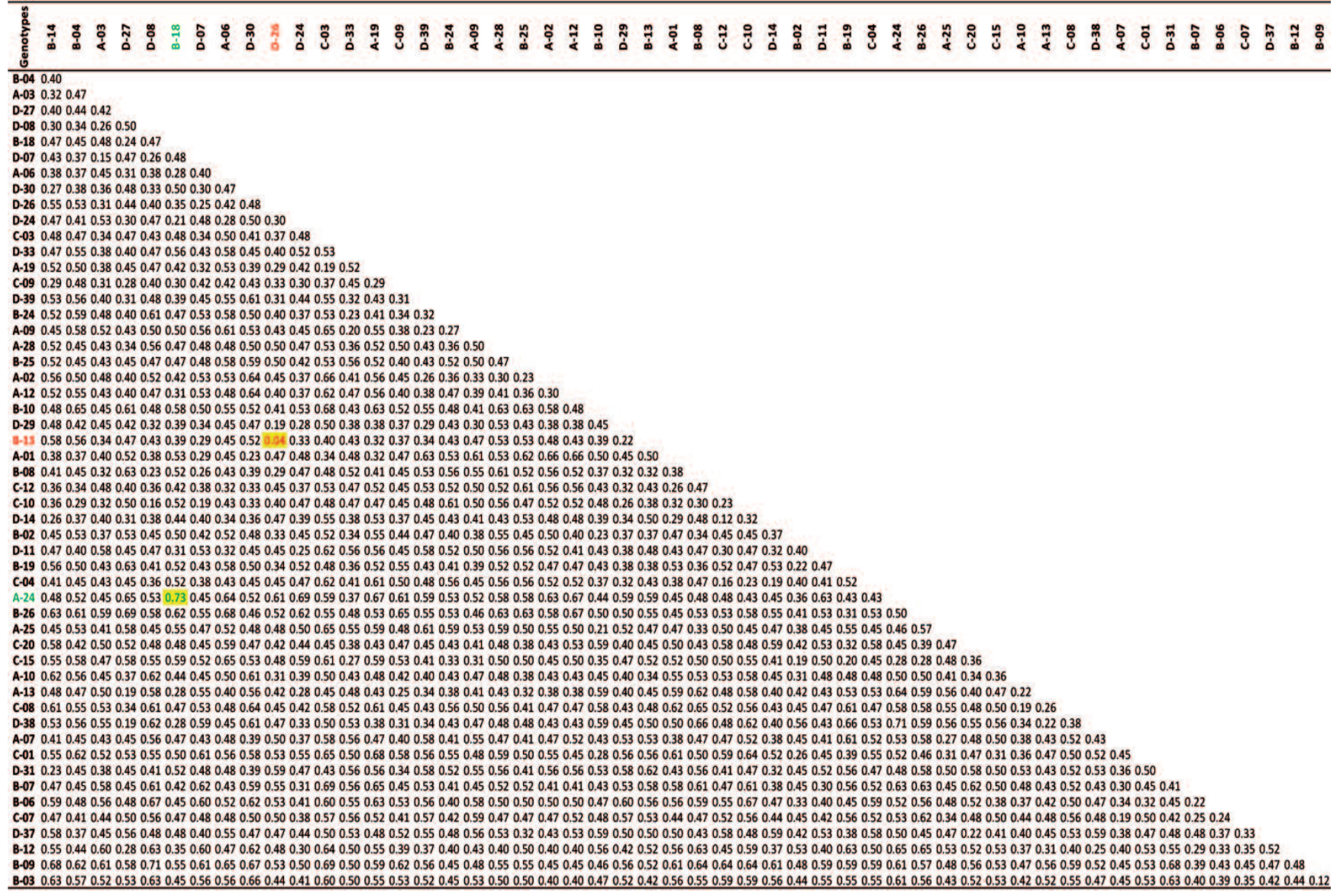

$$
\mathrm{Ib}=1-[2 \mathrm{x}|0.5-\mathrm{p}|]
$$

where, $\mathrm{p}$ is the proportion of all the accessions containing the fragment.

Genetic dissimilarity was calculated based on Jaccard's dissimilarity index using the software DARwin ver 5.0.158 (PERRIER and JACQUEMOUd-COLLET, 2006) where " 0 " and " 1 " were standardized as the least and maximum dissimilarity respectively. The dissimilarity matrix was used for tree construction following hierarchial clustering method using UPGMA algorithm implemented in the software DARwin ver 5.0.158. Confidence limits of different clades were tested by bootstrapping 1000 times to assess the repetitiveness of genotype clustering (FELSENSTEIN, 85).

The population genetic structure was inferred by analysis of variance (AMOVA) framework according to EXCOFFIER et al. (1992) using the Arlequin software version 3.11 (EXCOFFIER et al., 2005). The type of hierarchial AMOVA implemented here was with genotypic data, one group of populations and number within individual level. This technique treats genetic distances as deviations from a group mean position, and uses squared deviations as variances. The total sum of squares of genetic distances can then be partitioned into components that represent the within population and among population mean squares. AMOVA was conducted by grouping the genotypes on the basis of four collection sites and resin yield.

\section{Results}

Marker analyses

SSR analysis

A total of nineteen out of eighty initially screened SSR markers were selected on the basis of polymorphism. PCR amplification of the $P$. roxburghii genotypes using nineteen SSR markers produced a total of 46 bands, out of which 40 were polymorphic. The total number of polymorphic bands amplified per marker varied from 1 (pdms011, pdms221, pm07, RPtest6, RPtest9) to 4 (Pt30204 and Pt45002), with an average of 2.10 per marker (Table 2). Genetic divergence in terms of per cent polymorphism ranged from 50 to $100 \%$ with an average of $85.79 \%$ per marker.

\section{ISSR analysis}

A total of nine ISSR markers (University of British Columbia (UBC), Biotechnology laboratory, Vancouver, BC, Canada), selected from twenty-five initially screened markers, were used to evaluate the level of genetic variation among the high and low resin yielding genotypes of $P$. roxburghii. The primers demonstrated a 
comparable degree of polymorphism across the genotypes. PCR amplification using ISSR markers produced a total of 162 bands, out of which 148 were polymorphic. The total number of bands amplified per marker varied from 8 (UBC820) to 28 (UBC873), with an average of 16.44. Genetic divergence in terms of per cent polymorphism ranged from $33.33 \%$ (UBC880) to $100 \%$ (UBC811, UBC818, UBC846 and UBC873) with a mean of $84.59 \%$ per marker (Table 3 ).

\section{AFLP analysis}

A total of five out of twelve initially screened AFLP primer combinations were selected, on the basis of polymorphism and clarity of bands. PCR amplification of the $P$. roxburghii genotypes using AFLP markers produced a total of 250 bands, out of which ninety were found to be polymorphic. The total number of polymorphic bands amplified per marker varied from $6\left(\mathrm{E}_{\mathrm{AAG}} \mathrm{M}_{\mathrm{CTAC}}\right)$ to 43 $\left(\mathrm{E}_{\mathrm{ACA}} \mathrm{M}_{\mathrm{CTGC}}\right)$, with an average of 18 (Table 4). Genetic

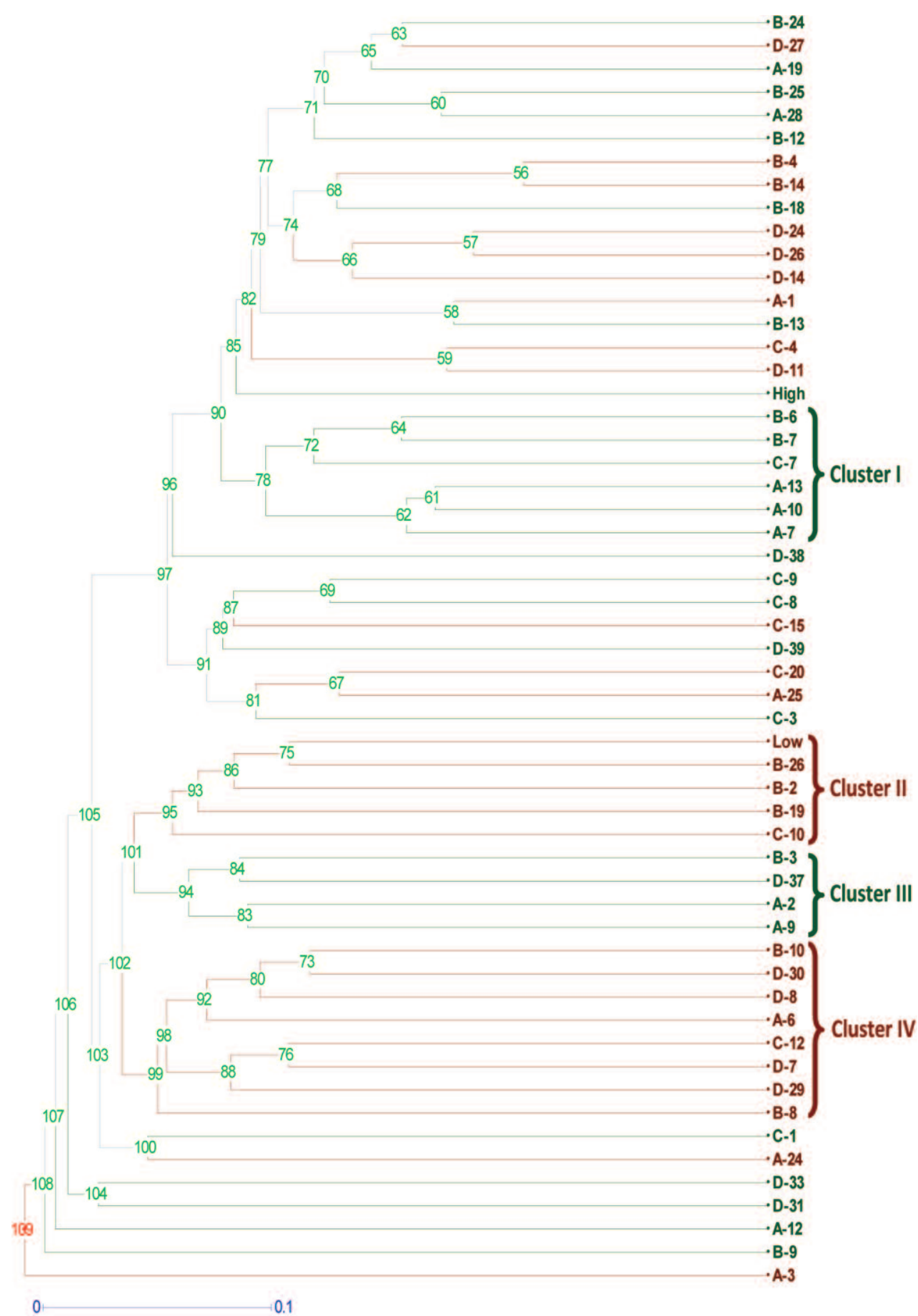

Figure 2. - Dendrogram showing genetic relationship among P. roxburghii genotypes varying in resin yield using ISSR markers. 


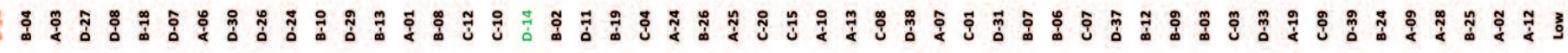

divergence in terms of per cent polymorphism ranged from $18.87 \%\left(\mathrm{E}_{\mathrm{ACA}} \mathrm{M}_{\mathrm{CTA}}\right)$ to $57.33 \%\left(\mathrm{E}_{\mathrm{ACA}} \mathrm{M}_{\mathrm{CTGC}}\right)$ with an average of $32.47 \%$ per marker.

\section{Cluster analysis}

Cluster analysis using SSR markers

The hierarchial clustering using UPGMA implemented in the software DARwin ver 5.0.158 revealed the existence of two distinct major clusters: Cluster-I and Cluster-II (Fig. 1). Cluster-I with a bootstrap value of 102, grouped twenty-eight genotypes together, out of which twenty-two were low resin yielders $(<3 \mathrm{~kg} /$ year $)$ and only six genotypes had high resin yield (>3 kg/year). Cluster-II with a bootstrap value of 101 , grouped twenty-five genotypes, out of which twenty-two had high resin yield and only three genotypes were low resin yielders.

The genetic dissimilarity index revealed high genetic diversity among the fifty-three genotypes of $P$. roxburghii used in the study. The dissimilarity coefficients ranged from 0.04 to 0.72 . The genotypes D-26 (2.1 kg/year) and B-13 (1.7 kg/year) had low resin yield and were found to be most similar, whereas A-24 (2.8 $\mathrm{kg} /$ year) and B-18 (3.35 kg/year) were found to be the most dissimilar genotypes (SI-1).

\section{Cluster analysis using ISSR markers}

The hierarchial clustering using UPGMA algorithm implemented in the software DARwin ver 5.0.158 did not revealed any distinct major clusters. However, four small clusters could be identified in which the grouping was according to the resin yield (Fig. 2). Since the resin yield of genotypes varied between 0.25 and $8 \mathrm{~kg} /$ year with an average of $3 \mathrm{~kg} / \mathrm{year}$, therefore the genotypes with resin yield below average $(<3 \mathrm{~kg} /$ year $)$ were considered to be low resin yielders and those with resin yield above average (>3 kg/year) were considered as high resin yielders. Cluster-I grouped six genotypes together (B-6, B-7, C-7, A-13, A-10 and A-7) with a bootstrap value of 78. All of these genotypes had high resin yield (above $3 \mathrm{~kg} /$ year). Cluster-II with a bootstrap value of 95 grouped five genotypes together (pooled DNA of low resin yielders, B-26, B-2, B-19 and C-10) all of which had low resin yield (below $3 \mathrm{~kg} /$ year). Cluster-III grouped four high resin yielders together (B-3, D-37, A-2 and A-9) with a bootstrap value of 94. Cluster-IV grouped eight low resin yielders together (B-10, D-30, D-8, A-6, C-12, D-7, D-29 and B-8) with a bootstrap value of 99 .

The genetic dissimilarity index calculated between samples ranged from 0.21 to 0.73 suggesting a high 
genetic diversity among the genotypes of $P$. roxburghii used in the study. The genotypes B-4 $(0.8 \mathrm{~kg} / \mathrm{year})$ and B-14 $(0.25 \mathrm{~kg} /$ year $)$ both having low resin yield were found to be most similar. On the other hand, the genotypes B-3 (4.9 kg/year) and D-14 (2.3 kg/year) were found to be the most dissimilar from each other (SI-2).

Cluster analysis using AFLP markers

The UPGMA based dendrogram revealed three broad clusters, where the genotypes clustered together on the basis of resin yield (Fig. 3). Cluster-I with a bootstrap value of 89 grouped twenty-four genotypes together out of which nineteen were high resin yielders ( $>3 \mathrm{~kg} / \mathrm{year})$ and only five genotypes had low resin yield ( $<3 \mathrm{~kg} /$ year). Cluster-II with a bootstrap value of 88 grouped eleven genotypes all of which had low resin yield except B-18. Cluster-III with a bootstrap value of 92 grouped seven genotypes together all of which were low resin yielders except B-13.

The genetic dissimilarity index was generated for $P$. roxburghii genotypes. The dissimilarity coefficient ranged from 0.01 to 0.22 suggesting a low genetic diversity among the genotypes of $P$. roxburghii used in the study. The genotypes A-7 (4.1 kg/year) and B-9 (4.7 kg/year) had high resin yield and were found to be most similar, whereas B-8 (2.2 kg/year) and D-24 $(1.7 \mathrm{~kg} /$ year) were found to be the most dissimilar genotypes (SI-3).

\section{Comparison of marker systems}

Four main aspects of the performance of the examined marker systems were considered: overall efficiency of polymorphism detection in the germplasm (i.e. PIC), the

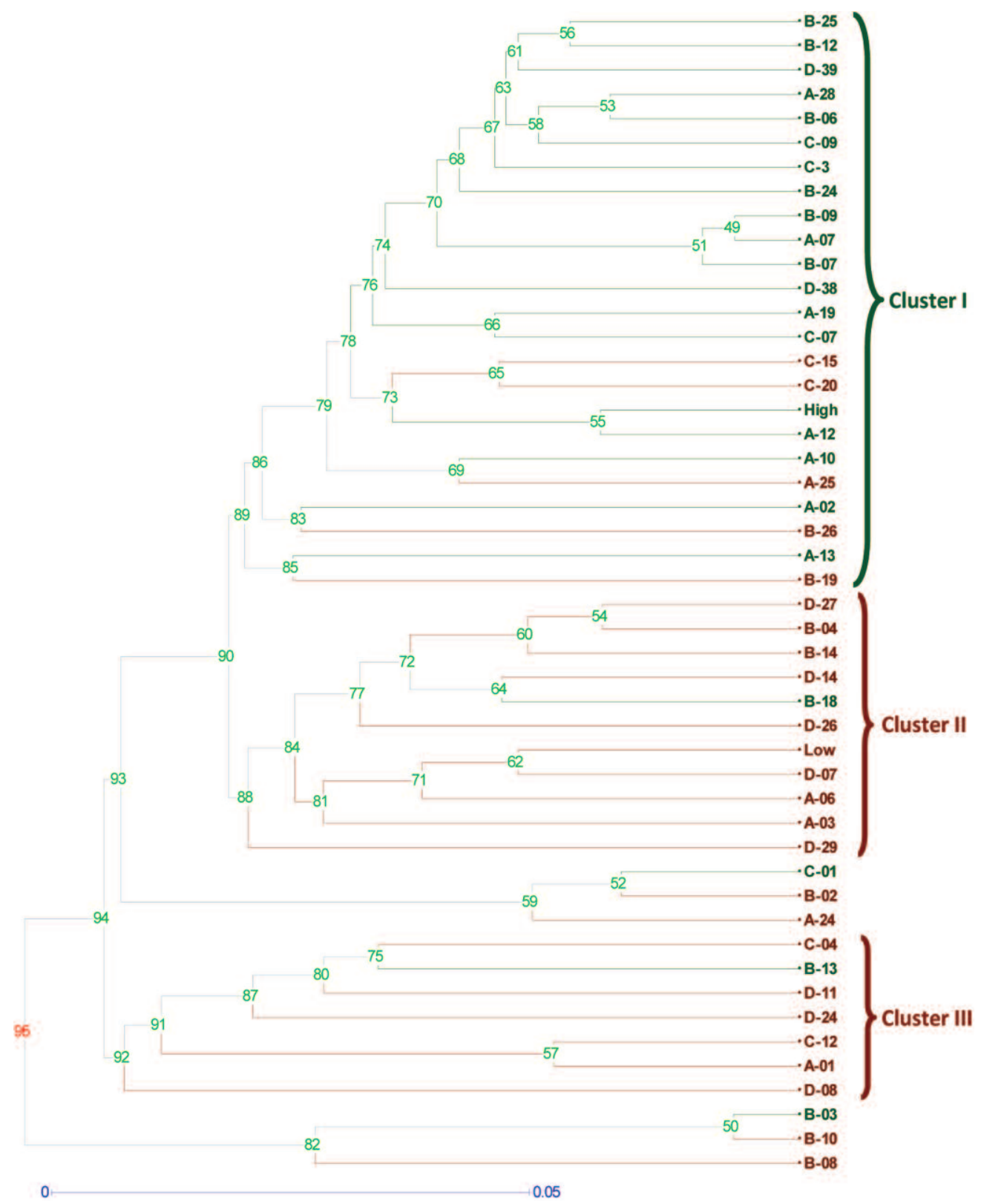

Figure 3. - Dendrogram showing genetic relationship among $P$. roxburghii genotypes varying in resin yield using AFLP markers. 


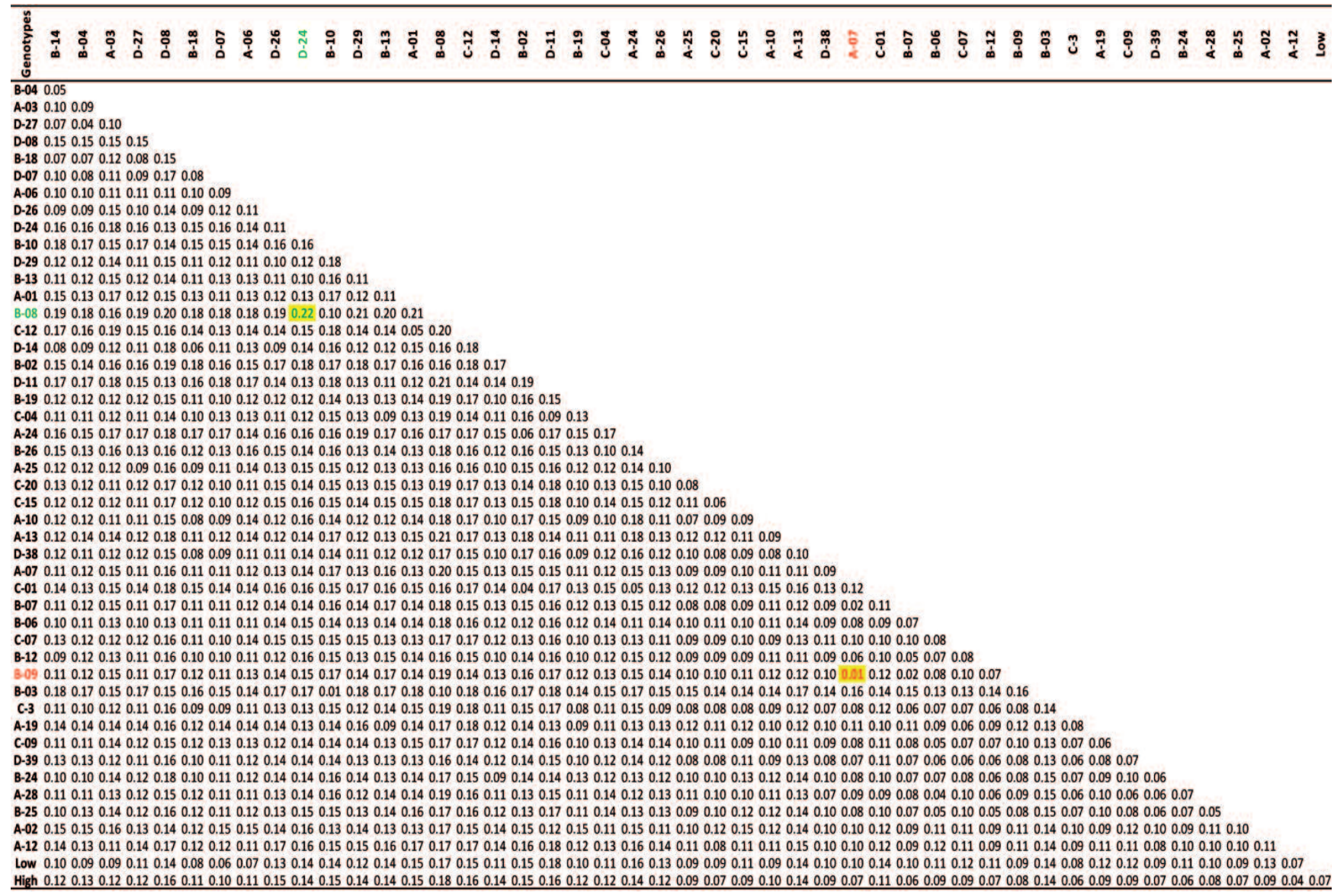

number of polymorphic loci assayed simultaneously (called effective multiplex ratio), the overall utility of a marker system for detecting genetic variation or marker index and the resolving power of a given marker system (Table 5).

\section{Polymorphism information content (PIC)}

SSR markers showed highest level of marker polymorphism in the examined germplasm as the PIC value for the SSR markers ranged from 0.100 (RPtest 9) to 0.499 (Pt 71936) with an average of 0.327 per marker (Table 2 ). There was a strong correlation between polymorphism and PIC $\left(r^{2}=0.864\right)$. AFLP markers detected lowest level of polymorphism with the PIC value ranging from $0.045\left(\mathrm{E}_{\mathrm{AAG}} \mathrm{M}_{\mathrm{CTAC}}\right)$ to $0.196\left(\mathrm{E}_{\mathrm{ACA}} \mathrm{M}_{\mathrm{CTGC}}\right)$ with an average of 0.095 per marker (Table 4). There was a strong correlation between polymorphism and PIC $\left(r^{2}=0.996\right)$. ISSR markers had PIC value ranging from 0.064 (UBC880) to 0.406 (UBC818) with an average of 0.289 per marker (Table 3) showing an intermediate level of polymorphism. There was a strong correlation between polymorphism and PIC $\left(r^{2}=0.827\right)$.

\section{Effective multiplex ratio (EMR)}

Effective multiplex ratio was highest for ISSR markers ranging from 1 (UBC 880) to 28 (UBC 873) with an average of 15.536 per marker. SSR markers showed the lowest EMR ranging from 0.5 (RP test 9) to 4 (Pt 30204) with an average of 1.932 per marker. AFLP markers showed intermediate values for EMR ranging from 1.28 $\left(\mathrm{E}_{\mathrm{AAG}} \mathrm{M}_{\mathrm{CTAC}}\right)$ to $24.65\left(\mathrm{E}_{\mathrm{ACA}} \mathrm{M}_{\mathrm{CTGC}}\right)$ with an average of 7.784. Although, AFLP markers produce a large number of fragments but the EMR depends on the fraction of polymorphic fragments and many of the fragments obtained were monomorphic across the examined genotypes.

\section{Marker index (MI)}

ISSR markers showed the highest marker index ranging from 0.064 (UBC880) to 9.746 (UBC818) with an average of 4.958 per marker (Table 3). There was a strong correlation between polymorphism and MI $\left(\mathrm{r}^{2}=0.835\right)$, and MI was positively correlated with PIC $\left(r^{2}=0.655\right)$. SSR markers showed the lowest values for marker index ranging from 0.050 (RPtest9) to 1.168 (Pt30204) with an average of 0.695 per marker (Table 2). There was a strong correlation between polymorphism and MI $\left(r^{2}=0.942\right)$, and MI was positively correlated with PIC $\left(r^{2}=0.822\right)$. AFLP markers had intermediate values for marker index ranging from $0.058\left(\mathrm{E}_{\mathrm{AAG}}\right.$ $\left.\mathrm{M}_{\mathrm{CTAC}}\right)$ to $4.824\left(\mathrm{E}_{\mathrm{ACA}} \mathrm{M}_{\mathrm{CTGC}}\right)$ with an average of 1.238 per marker (Table 4). There was a strong correlation between polymorphism and MI $\left(r^{2}=0.884\right)$, and MI was positively correlated with PIC $\left(r^{2}=0.919\right)$. 
Table 5. - Comparison of SSR, ISSR and AFLP marker systems.

\begin{tabular}{|c|c|c|c|c|c|c|c|}
\hline $\begin{array}{c}\text { Marker } \\
\text { system }\end{array}$ & $\begin{array}{c}\text { No. of primer } \\
\text { pairs } \\
\text { analyzed }\end{array}$ & $\begin{array}{c}\text { No. of loci } \\
\text { amplified }\end{array}$ & $\begin{array}{c}\text { Fraction of } \\
\text { polymorphic } \\
\text { markers }(\boldsymbol{\beta})\end{array}$ & $\begin{array}{c}\text { Average } \\
\text { PIC }\end{array}$ & $\begin{array}{c}\text { Effective } \\
\text { multiplex ratio } \\
(\mathrm{E}=\mathbf{n} \times \boldsymbol{\beta})\end{array}$ & $\begin{array}{c}\text { Marker } \\
\text { index (MI) }\end{array}$ & $\begin{array}{c}\text { Resolving } \\
\text { power } \\
(\mathbf{R P})\end{array}$ \\
\hline SSR & 19 & 46 & 0.86 & 0.33 & 1.93 & 0.695 & 1.164 \\
\hline ISSR & 9 & 162 & 0.84 & 0.29 & 15.53 & 4.960 & 8.060 \\
\hline NFIP & 5 & 250 & 0.36 & 0.09 & 7.78 & 1.238 & 8.099 \\
\hline
\end{tabular}

Table 6. - AMOVA by collection sites and resin yield using SSR, ISSR and AFLP marker systems.

\begin{tabular}{|c|c|c|c|c|c|}
\hline $\begin{array}{l}\text { Marker } \\
\text { system }\end{array}$ & $\begin{array}{c}\text { No. of } \\
\text { accessions }\end{array}$ & Categories & $\begin{array}{l}\text { Variation among } \\
\text { populations }\left(\mathbf{F}_{S T}\right)\end{array}$ & $\begin{array}{l}\text { Variation within } \\
\text { populations }\end{array}$ & p-value \\
\hline \multirow[t]{2}{*}{ SSR } & 53 & Collcction siles ${ }^{\mathrm{at}}$ & 0.0037 & 99.62 & \\
\hline & & Resin yicld ${ }^{b}$ & 0.1096 & 89.04 & $P<0.001$ \\
\hline \multirow[t]{2}{*}{ ISSR } & 53 & Collection sites ${ }^{\mathrm{a}}$ & 0.0058 & 99.41 & \\
\hline & & Resin yield ${ }^{\mathrm{h}}$ & 0.0483 & 95.17 & $P<0.001$ \\
\hline \multirow[t]{2}{*}{ AFL,P } & 46 & Collection sites ${ }^{\mathrm{a}}$ & 0.0491 & 95.09 & \\
\hline & & Resin yield ${ }^{b}$ & 0.2422 & 75.77 & $P<0.001$ \\
\hline
\end{tabular}

The type of hierarchial AMOVA implemented here was with genotypic data, one group of populations and no. within individual level.

${ }^{a}$ collection sites: four sites differing in altitude, aspect and site quality.

${ }^{\mathrm{b}}$ resin yield: resin yield was grouped as low (below $2.5 \mathrm{~kg}$ ), medium $(2.5-3.0 \mathrm{~kg}$ ) and high (above $3.0 \mathrm{~kg}$ ).

\section{Resolving power (RP)}

Since AFLP markers produce a large number of bands so they had the highest resolving power ranging from as low as $1.644\left(\mathrm{E}_{\mathrm{AAG}} \mathrm{M}_{\mathrm{CTAC}}\right)$ to $22.202\left(\mathrm{E}_{\mathrm{ACA}} \mathrm{M}_{\mathrm{CTGC}}\right)$ with an average value of 8.099 per marker (Table 4). There was a strong correlation between polymorphism and RP $\left(r^{2}=0.965\right)$ and $\mathrm{RP}$ was positively correlated with PIC $\left(r^{2}=0.984\right)$. SSR markers showed low resolving power as compared to ISSR and AFLP ranging from 0.226 (RPtest9) to 1.926 (Pt30204) with an average of 1.164 per marker (Table 5). There was a strong correlation between polymorphism and $\mathrm{RP}\left(\mathrm{r}^{2}=0.725\right)$ and $\mathrm{RP}$ was positively correlated with PIC $\left(\mathrm{r}^{2}=0.712\right)$. ISSR markers showed intermediate resolving power ranging from 0.679 (UBC880) to as high as 14.407 (UBC818) with an average of 8.059 per marker. There was a strong correlation between polymorphism and $\mathrm{RP}\left(\mathrm{r}^{2}=0.838\right)$, and $\mathrm{RP}$ was positively correlated with PIC $\left(r^{2}=0.759\right)$.

\section{Partitioning of variance}

As fixation indices $\left(\mathrm{F}_{S T}\right)$ measure the amount of differentiation among sub-populations derived from the subdivision of an original population (WRIGHT, 1978), values for $\mathrm{F}_{S T}$ range from 0 for non-differentiation to 1 for complete differentiation between an original population and its subpopulations, respectively. The qualitative guidelines for the interpretation of $\mathrm{F}_{S T}$ as suggested by WRIGHT (1978) ranging from 0 to 0.05 can be considered for an indication of little genetic differentiation. The values of $\mathrm{F}_{S T}$ from 0.05 to $0.15,0.15$ to 0.25 and above 0.25 indicate moderate, great and very great genetic differentiation, respectively. In this study, AMOVA by collection site using all the three marker systems revealed $\mathrm{F}_{S T}$ values less than 0.05 indicating little genetic differentia- tion and they were not found to be significant for all the three marker systems. On the other hand, in case of AMOVA by resin yield, $\mathrm{F}_{S T}$ values were $0.1096,0.0483$ and 0.2422 for SSR, ISSR and AFLP marker systems respectively, representing moderate, little and great genetic differentiation. The $\mathrm{F}_{S T}$ values for all the three marker systems in case of AMOVA by resin yield were found to be highly significant at $\mathrm{p}<0.001$ (Table 6).

\section{Discussion}

A variety of molecular markers have been used for estimating the genetic diversity in different Pinus species such as RAPD (GOMEZ et al., 2001; NKONGOLO et al., 2002; PENG et al., 2003; KANDEDMIR et al., 2004; KIM et al., 2005; NAUGZEMYS et al., 2006;), ISSR (HoNG et al., 2004; Hong et al., 2007; RANGER et al., 2008; ZHANG et al., 2005; PARASharmi and Thengane, 2012), AFLP (MARIETTE et al., 2001; LERCETEAU and SzMIDT, 1999) and SSR (Provan et al., 1998; Provan et al., 1999; Boys et al., 2005). The use of a particular molecular marker type for estimating the genetic diversity of germplasm collections however depends on many factors including costs on genotyping the large populations with a marker assay (GUPTA et al., 2002). In recent years, the SSR and SNP markers derived from ESTs, due to their inexpensive developmental costs (KOTA et al., 2003; VARSHNEY et al., 2005), are increasingly being used for genotyping of natural or inbreeding populations. Together with these markers, AFLP and ISSR markers are still considered good for fingerprinting or diversity analysis. Therefore, the present study documents the comparative utility of these marker types for molecular characterization of genotypes varying for a trait. 


\section{Marker polymorphism}

SSR markers were found to be highly polymorphic while AFLP markers showed a lower level of polymorphism with ISSR markers showing intermediate level of polymorphism in the germplasm examined in present study. This relates to the variation of the average number of polymorphic bands per assay unit, which ranged from a minimum of $32.47 \%$ for AFLP markers and $84.59 \%$ for ISSR markers to a maximum of $85.79 \%$ for SSR markers. The higher level of polymorphism detected by SSR markers than with ISSRs and AFLPs highlights the discriminating capacity of the former. The hypervariability observed at SSR loci was expected because of the unique mechanism by which this variation is generated: replication slippage is thought to occur more frequently than single nucleotide mutations and insertion/deletion events, which generate the polymorphisms detectable by AFLP (PowELL et al., 1996). Cent per cent polymorphism was observed for SSR as well as ISSR markers. This has also been reported previously by different researchers (MARAS et al., 2008; CHEZHiAn et al., 2009; BAGHIZADEH et al., 2010).

A comparison of the overall efficiency of the three marker systems was provided by the marker index. MI was highest (4.958) for ISSR markers, lowest (0.695) for SSR markers and intermediate (1.238) for AFLP marker systems. Almost seven-fold higher MI calculated for ISSR and two-fold higher MI for AFLP markers in comparison to SSR highlights the distinctive nature of the ISSR and AFLP assay, which is in concurrence with earlier reports in many plant species [soybean (PowELL et al., 1996); Iranian Pistachio (BELAJ et al., 2003); rice (SAINI et al., 2004); wheat (MEDINI et al., 2005); olive (BAGHIZADEH et al., 2010)]. The distinctive value of MI for ISSR and AFLP data is related to the effective multiplex ratio. In other words, it depends more on the high number of polymorphic bands obtained per experiment than on the allelic heterozygosity found among accessions. This feature makes the ISSR and AFLP markers suitable for fingerprinting or estimating genetic diversity in breeding populations. In case of AFLPs, although 5 out of 6 primer combinations were polymorphic, these markers showed low level of polymorphism in comparison to ISSR markers in the germplasm collection examined. In the present study, the portion of AFLP bands/loci that met the quality criteria for scoring was low. However, the resolution of AFLP fragments also depends on the fragment detection system. The EMR was lowest for SSR markers but they showed highest level of polymorphism as reported in earlier studies (BAGHIZADEH et al., 2010). This state could be attributed to the lower number of bands per assay detected by SSR markers.

\section{Comparative utility of different marker systems}

Assessment of genetic diversity by using molecular markers is important not only for tree improvement efforts but also for efficient management and conservation of plant genetic resources in genebanks (GRANER et al., 2004). Therefore, the selection of a particular type of molecular marker is important and critically depends on the intended use (GUPTA et al., 2002).
Regarding the detection of polymorphism, SSR markers certainly are better than ISSR or AFLP markers as SSR are multi allelic markers in contrast to ISSR or AFLP markers. Because of detecting higher polymorphism, SSR markers showed low genetic similarity among the genotypes ranging from 0.409 to 0.955 suggesting high genetic variability among the genotypes. This was followed by ISSR markers showing genetic similarity ranging from 0.537 to 0.858 and AFLP markers showing highest genetic similarity ranging from 0.79 to 0.984 among the genotypes. This is in agreement with other studies comparing the level of polymorphism detected with AFLP and SSR markers in soybean (Powell et al., 1996), maize (PEJIC et al., 1998), sorghum (MENZ et al., 2004), and triticale (TAMs et al., 2005). Posselt et al. (2006) also found low genetic similarity using SSR markers in comparison to ISSR and AFLP markers. This characteristic attribute of SSR markers together with their co-dominance nature made them the markers of choice in plant genetics and breeding (GUPTA and VARSHNEY, 2000; VARSHNEY et al., 2005). ISSR and AFLP are bi-allelic and less informative than SSRs.

Because of assaying one genetic locus per primer pair, the effective multiplex ratio for SSR markers was low (1.932). However, ISSR markers showed highest effective multiplex ratio as 15.536. This was due to the higher number of DNA fragments amplified along with a higher proportion of polymorphic bands. Although, AFLP markers produced the highest number of bands but the proportion of polymorphic bands was lower as compared to ISSR markers so AFLP markers showed intermediate (7.784) effective multiplex ratio.

\section{Relationships in examined germplasm}

As expected the grouping of genotypes on the basis of resin yield was more distinct with SSR markers as compared to ISSR and AFLP markers. The reason for this observation may be that the SSRs are highly specific and are co-dominant in nature thereby being capable of detecting heterozygous condition whereas, ISSR and AFLP markers are dominant and less polymorphic as compared to SSR markers.

\section{Analysis of molecular variance}

The results of cluster analysis were supported by the AMOVA analysis. Cluster analysis grouped the individuals on the basis of resin yield and not their site of collection. AMOVA by collection site suggested that the variation among the groups was not significant using all the three marker systems whereas AMOVA by resin yield revealed that the variation among the groups was highly significant for all the three marker systems. AFLP markers showed highest genetic differentiation among the groups followed by SSR and ISSR markers showing moderate and little genetic differentiation respectively. This clearly indicated that the genetic variation among the genotypes is not related to their site of collection in terms of altitude and aspect but it correlates with the variation in resin yield. AMOVA by resin yield using all the three marker systems revealed the variation among groups to be highly significant. Similar analysis was 
performed by CHO et al., 2008 in Soybean in which AMOVA by usage group, AMOVA by collection sites and AMOVA by maturity groups revealed the variation among groups to be highly significant.

\section{Conclusion}

The present study highlights the advantages and disadvantages of different marker systems for diversity analysis in natural populations to exploit the genotyping data for tree improvement. For estimating the genetic diversity of germplasm collections, ISSR or AFLP markers are more suitable as they have higher marker index. SSR markers can be used for such analyses (especially, if more than one marker can be used by multiplexing). As far as the relationship among genotypes based on some specific trait is concerned, SSR markers would prove to be better than ISSR and AFLP markers. The results of this study suggest that the genotypes of $P$. roxburghii varying in resin yield can be better differentiated using SSR and AFLP markers or a combination of the two. Characterization of the $P$. roxburghii genotypes varying in resin yield will help to provide more insights into the genetic relationships among them and also to explore associations of molecular markers with the resin yielding trait which will be helpful for tree improvement and marker assisted breeding in $P$. roxburghii.

\section{References}

AsIF, M. J. and C. H. CANNON (2005): DNA Extraction from Processed Wood: A case study for the identification of an endangered timber species (Gonystylus bancanus). Plant Molecular Biology Reporter, 23: 185-192.

BAghizAdeH, A., S. Noroozi and M. J. JaVARAN (2010): Study on genetic diversity of some Iranian Pistachio (Pistacia vera L.) cultivars using random amplified polymorphic DNA (RAPD), inter simple sequence repeat (ISSR) and simple sequence repeat (SSR) markers: A comparative study. African Journal of Biotechnology, 9(45): 7632-7640.

Belaj, A., Z. SAtovic, G. Cipriani, L. Baldoni, R. Testolin, L. RAllo and I. Trujillo (2003): Comparative study of the discriminating capacity of RAPD, AFLP and SSR markers and of their effectiveness in establishing genetic relationships in olive. Theoretical and Applied Genetics, 107: 736-744.

Boys, J., M. Cherry and S. DAYANANDAN (2005): Microsatellite analysis reveals genetically distinct populations of Red Pine (Pinus resinosa, Pinaceae). American Journal of Botany, 92(5): 833-841.

Chauhan, P., H. S. Ginwal, A. RAwat and S. Barthwal (2010): Cross-species amplification and characterization of chloroplast and nuclear microsatellite markers in Himalayan Chir Pine (Pinus roxburghii Sarg.) Molecular Ecology Resources, 11: 219-222.

Chauhan, K. C. and M. S. KANWAR (2001): Nature of variability and character associations for cone and seed characteristics in Pinus roxburghii Sargent plus trees. Indian Journal of Genetics and Plant Breeding, 61: 151-154.
Chezhian, P., R. Yasodha and M. Ghosh (2009): Genetic diversity analysis in a seed orchard of Eucalyptus tereticornis. New Forests, 85-99.

Cho, G. T., J. Lee, J. K. Moon, M. S. Yoon, H. J. BaeK, J. H. Kang, T. S. Kim and N. C. PAeK (2008): Genetic diversity and population structure of Korean soybean landrace [Glycine $\max (\mathrm{L}$.$) Merr.]. J. Crop Sci. Biotech.$ 11(2): 83-90

Coppen, J. J. W. and G. A. Hone (1995): Gum naval stores: turpentine and rosin from pine resin. FAO, Nonwood Forest Products, 2: $62 \mathrm{p}$.

DoGRA, P. D. (1985): Conifers of India and their wild gene resources in relation to tree breeding. Indian Forester, 111(11): 935-955.

Doyle, J. J and J. L. Doyle (1990): A rapid total DNA preparation procedure for fresh plant tissue. Focus, 12: 13-15.

Excoffier, L., G. LaVal and S. SchneIder (2005): Arlequin ver. 3.0: An integrated software package for population genetics data analysis. Evolutionary Bioinformatics online, 1: 47-50.

Excoffier, L., P. E. Smouse and J. M. Quattro (1992): Analysis of molecular variance inferred from metric distances among DNA haplotypes; Application to human mitochondrial DNA restriction data. Genetics, 131: 479-491.

Felsenstein, J. (1985): Confidence limits in phylogenies: an approach using bootstrap. Evolution, 39: 783-791.

Gauli, A., O. Gailing, V. M. Stefenon and R. Finkeley (2009): Genetic similarity of natural populations and plantations of Pinus roxburghii Sarg. in Nepal. Annals of Forest Science, 66: 7031-7039.

Ginwal, H. S., P. Chauhan, S. S. MAURYa and V. S. JAdON (India) (2010): Genetic variability in Pinus roxburghii Sarg. revealed by RAPD markers. Bioremediation, Biodiversity and Bioavailability, 4(1): 28-34.

Gomez, A., R. AliA and M. A. Bueno (2001): Genetic diversity of Pinus halepensis Mill. Populations detected by RAPD loci. Annals of Forest Science, 58: 869-875.

Graner, K. J., T. Dehmer and A. Thiel (2004): Borner, Plant genetic resources: benefits and implications of using molecular markers. In: M. C. DE VICENTE (Ed.), Issues in Genetic Resources No. 11, IPGRI, Rome, Italy, pp. 26-32.

Gupta, G. C., K. C. Sharma and C. Singh (1970): Programme for breeding of high resin yielders of Chir pine in Himachal Pradesh. In: Proceedings of seminar-cumworkshop on genetic improvement of forest tree seeds in India. Feb. 7-1, Dehradun, pp. 46-50.

Gupta, P. K. and R. K. VARshney (2000): The development and use of microsatellite markers for genetic analysis and plant breeding with emphasis on bread wheat. Euphytica, 113(23): 163-185

Gupta, P. K., R. K. VARShney and M. PRAsad (2002): Molecular markers: principles and methodology. In: S. M. JAIN, B. S. AhlOOWALIA, D. S. BRAR (Eds.), Molecular Techniques in Crop Improvement, Kluwer Academic Publishers, The Netherlands, 2002, pp. 9-54.

HoNG, Y. P., H. Y. KwoN and I. S. KIM (2007): I-SSR markers revealed inconsistent phylogeographic patterns among populations of Japanese Red Pines in Korea. Silvae Genetica, 56(1): 22-26.

Hong, Y. P., H. Y. K. Won, K. S. KIM, K. N. Hong and Y. Y. KIM (2004): Discordance between geographical distribution and genetic relationship among populations of Japanese red pines in Korea revealed by analysis of ISSR markers. Silvae Genetica, 53(3): 89-92. 
Hussain, A. (1995): Untersuchungen zur genetischen kontrolle von Isoenzyme-polymorphismen und zur genetischen Struktur von Pinus roxburghii Sarg. Thesis for the degree of Doctor of Forestry Science at the Faculty of Forestry Sciences and Forest Ecology, George-August University of Gottingen, Germany.

KANDEDMiR, G. E., I. KANDEDMIR and Z. KAYAL (2004): Genetic variation in Turkish Red Pine (Pinus brutia Ten.) seed stands as determined by RAPD markers. Silvae Genetica, 53: 4-5.

KIM, Y.Y., H. S. ChoI and B.Y. KANG (2005): An AFLPbased Linkage Map of Japanese Red Pine (Pinus densiflora) using haploid DNA Samples of mega-gametophytes from a single maternal tree. Molecules and Cells, 20(2): 201-209.

Kota, R., S. Rudd, A. Facius, G. Kolesov, T. Thiel, H. Zhang, N. Stein, K. MAYer and A. Graner (2003): Snipping polymorphisms from large EST collections in barley (Hordeum vulgare L). Molecular Genetics and Genomics, 270: 224-233.

Le Maitre, D. C. (1998): Pines in cultivation: a global view. In: D. M. RICHARDSON (Eds.), Ecology and biogeography of Pinus, Cambridge University Press, New York, USA. pp. 407-431.

Lerceteau, L. E. and A. E. Szmidt (1999): Properties of AFLP markers in inheritance and genetic diversity studies of Pinus sylvestris. Heridity, 82: 252-260.

LI, G., and C. F. QuLRos (2001): Sequence-related amplified polymorphism (SRAP), a new marker system based on a simple PCR reaction: its application to mapping and gene tagging in Brassica. Theor Appl Genet, 103: 455-461.

MALABADI, R. B. and K. NATARAJA (2007): Spatio-temporal accumulation of peroxidases and assessment of clonal identity of somatic seedlings by ISSR and RAPD in Pinus roxburghii. American Journal of Plant Physiology, 2(6): 356-366.

Maras, M., J. Sustar-Vozlic, B. Javornik and V. Meglic (2008): The efficiency of AFLP and SSR markers in genetic diversity estimation and gene pool classification of common bean (Phaseolus vulgaris L.) Acta agriculturae Slovenica, 91-1.

Mariette S., D. Chagne, S. Decroocq, G. G. Vendramin, C. Lalanne, D. Madura and C. Plomion (2001): Microsatellite markers for Pinus pinaster Ait. Annals of Forest Science, 58: 203-206.

Martin-Trillo, M. and J. M. Martinez-Zapater (2002): Growing up fast: Manipulating the generation time of trees. Current Opinion in Biotechnology, 13: 151-155.

Medini, M., S. Hamza, A. Rebai and M. Baum (2005): Analysis of genetic diversity in Tunisian durum wheat cultivars and related wild species by SSR and AFLP markers. Genetic Resources and Crop Evolution, 52: 21-31.

Menz, M. A., R. R. Klein, N. C. Unruh, W. L. Rooney, P. E. KLEIN and J. E. Mullet (2004): Genetic diversity of public inbreds of Sorghum determined by mapped AFLP and SSR markers. Crop Science, 44: 1236-1244.

Naugzemys, D., D. Zvinila, A. Aucina and V. Rancelis (2006): Comparison of DNA polymorphism in seedlings of Pinus sylvestris L. from different populations by RAPD markers. Biologia, 1: 30-35.

Nautiyal, S., M. Tyagi, S. S. Negi, R. K. SRivastava and S. NAUTIYAL (2006): Improvement of Chir pine (Pinus roxburghii Sarg.). In: (Eds.) Studies on Himalayan Pines. Pub. FRI, Dehradun, India. pp. 50-63.
Nkongolo, K. K., P. Michael and W. S. Gratton (2002): Identification and characterization of RAPD markers inferring genetic relationships among Pine species. Genome, 45: 51-58.

Parasharami, V. A. and S. R. Thengane (2012): Inter population genetic diversity analysis using ISSR markers in Pinus roxburghii (Sarg.) from Indian provenances. International Journal of Biodiversity and Conservation, 4(5): 219-227.

Pejic, I., P. AJmone-Marsan, M. Morgante, V. Kozumplick, P. Castiglioni, G. TARAmino and M. Motto (1998): Comparative analysis of genetic similarity among maize inbred lines detected by RFLPs, RAPDs, SSRs, and AFLPs. Theoretical and Applied Genetics, 97: 1248-1255.

Peng, S. L., Q. F. LI, D. LI, Z. F. WANG and D. P. WANG (2003): Genetic diversity of Pinus massoniana revealed by RAPD markers. Silvae Genetica, 52(2): 60-63.

PerRier, X. and J. P. JACQUemoud-Collet (2006): DARwin software. Genetic improvement of vegetatively propagated crops. http://darwin.cirad.fr/Home.php

Posselt, U. K., P. Barre, G. Brazauskas and E. B. TURNER (2006): Comparative Analysis of genetic Similarity between Perennial Ryegrass Genotypes Investigated With AFLPs, ISSRs, RAPDs and SSRs. Czech J. Genet. Plant Breeding, 42(3): 87-94.

Powell, W., G. MachraY and J. Provan (1996): Polymorphism revealed by simple sequence repeats. Trends in Plant Sciences, 1: 215-222.

Prevost, A. and M. Wilkinson (1999): A new system of comparing PCR primers applied to ISSR fingerprinting of potato cultivars. Theoretical and Applied Genetics, 98: 107-112.

Provan, J., N. Soranzo, N. J. Wilson, D. B. Goldstein and W. Powell (1999): A low mutation rate for chloroplast microsatellites. Genetics, 153: 943-947.

Provan, J., N. Soranzo, N. J. Wilson, J. W. McNicol, G. I. Forrest, J. Cotrell and W. Powell (1998): Genepool variation in Caledonian and European Scots pine (Pinus sylvestris L.) revealed by chloroplast Simple Sequence Repeats. In: Proceedings of Royal Society of London, Series B, 265: 1697-1705.

Ranger, M., K. K. Nkongolo, P. Michael and P. Beckett (2008): Genetic differentiation of Jack Pine (Pinus banksiana) and Red Pine ( $P$. resinosa) populations from metal contaminated areas in northern Ontario (Canada) using ISSR markers. Silvae Genetica, 57(6): 333-340.

Roldan-Ruiz Dendauw, J., E. Van Bockstaele, A. Depicker and M. DE Loose (2000): AFLP markers reveal high polymorphic rates in ryegrasses (Lolium spp.). Molecular Breeding, 6: 125-134.

SAINI, N., N. JAIN, S. JAIN and R. K. JAIN (2004): Assessment of genetic diversity within and among Basmati and non-Basmati rice varieties using AFLP, ISSR and SSR markers. Euphytica, 140: 133-146.

SHARMA, K. (1999): Genetic variability and population structure of Pinus roxburghii Sarg. India. PhD. Thesis, FRI Deemed University Dehra Dun.

SHARMA, R. (2003): Studies on nursery and early field evaluation of provenance-cum-progeny test in Pinus roxburghii Sarg. PhD. Thesis, FRI Deemed University, Dehradun.

Sharma, R., S. Kumar and K. S. ThakuR (2006): Genetic improvement of Chir pine (Pinus roxburghii Sargent.) in India - A review. The Indian Forester, 132(3): 314-328. 